\title{
La biodiversidad de Colombia: significado y distribución regional
}

\author{
J. Orlando Rangel-Ch. ${ }^{1,2}$ \\ ${ }^{1}$ Instituto de Ciencias Naturales, Universidad Nacional de Colombia \\ ${ }^{2}$ Academia Colombiana de Ciencias Exactas, Físicas y Naturales
}

\begin{abstract}
Resumen
Con base en el modelo geográfico de regiones naturales de Colombia, a saber, costa Pacífica o Chocó biogeográfico, costa Caribe, Llanos (Orinoquia), Amazonía y Andina, se presentan los resultados de los inventarios de biodiversidad. En flora, se incluyen a las plantas con flores, a los helechos, a los musgos y a los líquenes; en la fauna a los reptiles, las aves, los anfibios y a los mamíferos y en la vegetación a las comunidades vegetales terrestres y acuáticas. Los análisis del clima se hicieron desde una perspectiva ecológica con tratamientos detallados de la precipitación. Los ecosistemas se definieron con base en las caracterizaciones de los componentes bióticos (especies, comunidades) y en los aspectos complementarios relacionados con la geología, el clima y los suelos. Los resultados muestran que a nivel del globo, Colombia es uno de los dos países con mayor expresión de este capital natural renovable (biodiversidad). En flora, se cuenta con registros cercanos a las 26.500 especies de plantas con flores que le colocan como el segundo país con mayor riqueza, después de Brasil. En helechos hay 1.600 especies, en musgos 976 y en líquenes 1.700, valores que le colocan como el país más rico en cada uno de estos grupos a nivel neotropical. La diversidad y la riqueza de los bosques y otros tipos de vegetación como selvas, matorrales, pastizales, rosetales de Colombia, alcanza cerca de 1.200 tipos diferentes, que le confieren característica singular como uno de los países con mayor variedad a nivel mundial. La región Andina o cordillerana es la que presenta mayor concentración de la biodiversidad; cerca de 11.500 especies de plantas con flores, 914 de musgos, 756 de hepáticas, 1.396 de líquenes y 1.050 de helechos, sirven de testimonio de la enorme concentración de la riqueza vegetal. En los grupos de fauna, los valores de 974 especies de aves, 484 de anfibios, 274 de reptiles y 349 de mamíferos, igualmente la posicionan en el primer lugar. Dos de nuestras regiones naturales más ricas, a saber, el Chocó biogeográfico o región Pacífica y el páramo, no tienen comparación en cuanto a su biodiversidad con otras regiones semejantes del mundo. A nivel de fauna, Colombia es el país más rico en aves (1.834 especies) y en anfibios (700 especies) y alcanza valores muy importantes en otros grupos como reptiles (512 especies) y mamíferos (492 especies).
\end{abstract}

Palabras clave: biodiversidad, flora, fauna, vegetación, ecosistemas, Colombia.

Biodiversity of Colombia: significance and regional distribution

\begin{abstract}
Based on the geographical pattern of natural regions of Colombia, namely Pacific coast - biogeographical Chocóregion, Caribbean coast, llanos (Orinoco), Amazon and Andean, results of inventories of biodiversity are presented. Flora includes flowering plants, ferns, mosses and lichens. Fauna include reptiles, birds, amphibians and mammals. Plant vegetation includes the terrestrial and aquatic plant communities. The climatic analysis was made from an ecological perspective with detailed analysis of precipitation; ecosystems were defined based on characterizations of biotic components (species, communities) and the complementary aspects related to geology, climate and soils. At the level of the world, Colombia is one of two countries with the highest expression of this renewable natural capital (biodiversity). In flora, it means around 26,500 species of flowering plants. There are 1,600 species of ferns, 976 mosses and 1,700 lichens, these values placed the country as the richest in each of these groups of neotropical countries. The diversity and richness of forests and other vegetation types as shrublands, grasslands, rosette-plants, Colombia reaches about 1,200 different types, which give unique feature as one of the countries with the greatest variety worldwide. The Andean region has the largest concentration of biodiversity; about 11,500 species of flowering plants, 914 mosses, 756 liverworts, lichens 1,396 and 1,050 of ferns, serve as testimony to the enormous concentration of plant richness; in faunal groups, the values of 974 bird species, 484 amphibians, 274 reptiles and 349 mammals, also show this natural region in the first place. The Chocó region of Colombia is richer than the areas of the same biogeographical region in Central America and Ecuador. The Colombian paramo region represented $85 \%$ of the richness of flowering plants the entire biogeographical region and about $41 \%$ of the species are restricted in distribution to Colombia. In terms of fauna richness Colombia is the richest country in birds $(1,834$ species) and amphibians (700 species) and reached very important values in other groups like reptiles (512 species) and mammals (492 species).
\end{abstract}

Key words: Biodiversity, flora, fauna, vegetation, ecosystems, Colombia. 


\section{Introducción}

La biodiversidad es la variabilidad al interior del mundo viviente y se expresa según niveles de organización biológica: genes, especies, poblaciones, comunidades o ecosistemas que se encuentran en una porción geográfica del territorio, es decir una localidad, una región, un país o en el globo. La herramienta básica para conocer la biodiversidad son los inventarios o "asiento de los bienes y cosas pertenecientes a persona o comunidad, hecho con orden y precisión" (RAE, 2001). El inventario nos permitirá conocer la representación del capital natural o biodiversidad, donde se encuentra, cual es su estado de conservación y cuales son las posibilidades de uso sostenible. Bajo estas consideraciones, en 1991 iniciamos en asocio con el INDERENA la preparación del documento básico que llevaría Colombia a la cumbre "Río 1992" sobre el estado del conocimiento de la biodiversidad.

Desde un principio se seleccionaron cinco ejes temáticos sobre los cuales versaría el gran propósito del inventario de la biodiversidad: la vegetación, la flora, la fauna el clima y los ecosistemas. Los propósitos eran recuperar la información y generar bases de datos con lo pertinente a taxonomía, distribución y ecología; complementar las bases de datos con información gris o no disponible en documentos internos de trabajo (museos, herbarios, archivos de instituciones nacionales e internacionales) y generar información nueva y divulgar ese conocimiento. Para cumplir con este último objetivo se inició en1995 la publicación de la serie Colombia Diversidad Biótica con la aparición del primer numero, Centros de concentración de especies - Fauna (Rangel ed., 1995) y prosigue con la aparición del volumen XIV, la región de la Orinoquia (Rangel ed., 2014). En el inventario de la flora se incluyeron a las plantas con flores, los helechos, los musgos y a los líquenes. En la fauna a los reptiles, las aves, los anfibios y los mamíferos. En la vegetación las caracterizaciones y los registros se relacionan con las comunidades vegetales terrestres y acuáticas. Los análisis del clima se han hecho desde una perspectiva ecológica con análisis detallados de la precipitación. Los ecosistemas han sido definidos tomando como base los resultados de las caracterizaciones de los componentes ya mencionados, más aspectos complementarios relacionados con la geología y los suelos.

Desde 1992, hasta ahora el inventario se ha realizado bajo la coordinación del grupo de investigación Biodiversidad y Conservación del Instituto de Ciencias Naturales de la Facultad de Ciencias, Universidad Nacional de Colombia. Desde un principio se adoptó el modelo de regiones naturales de Colombia, Costa pacífica o Chocó biogeográfico, costa Caribe, Llanos (Orinoquia), Amazonía y Andina.

Correspondencia:

J. Orlando Rangel-Ch., jorangelc@unal.edu.co

Recibido: 14 de octubre de 2014

Aceptado: 15 de abril de 2015
En esta contribución se presenta el estado actual del conocimiento de la biodiversidad en estos cinco componentes (flora, fauna, tipos de vegetación - ecosistemas y clima) a nivel de país y en detalle para las regiones naturales del Chocó biogeográfico, el Caribe y la Orinoquia. En razón a que no se dispone de los volúmenes integradores sobre la biodiversidad de la Amazonía y de la región Andina, solamente se presentan los datos globales y en el caso de la región Andina se detalla la biodiversidad del páramo.

Se trata de responder interrogantes básicos relacionados con la expresión de la riqueza y diversidad a nivel regional con referencia especial al componente vegetal (flora, vegetación) y de documentar la existencia de patrones de distribución de la biodiversidad asociados con variaciones del clima en las series ecológicas hídrica o de pantano y topográfica o altitudinal.

\section{Metodología}

Los inventarios tienen un componente histórico por cuanto se ha recuperado la información de diversas fuentes de los últimos 200 años. Igualmente se han desarrollado numerosas exploraciones de campo a diferentes localidades del país. La suma de los registros de estos dos esfuerzos constituye un inventario global de nombres. Posteriormente la consulta con especialistas o las revisiones y confrontaciones con portales especializados de Internet según el grupo (plantas o animales) han permitido alcanzar un inventario revisado y actualizado. La literatura consultada cubre las diferentes temáticas (taxonomía, sistemática, ecología, antropología, arqueología, ingeniería forestal) y las escalas locales, regionales y globales. Estos inventarios constituyen la base para los análisis de riqueza y diversidad, los cuales generalmente se realizan a nivel taxonómico (jerarquías) y unidades fisiográficas o de terreno según el área natural en estudio, como se muestra en los volúmenes publicados sobre las regiones naturales en las cuales se han concluido los inventarios básicos (pacífica o Chocó biogeográfico, costa Caribe, llanos u Orinoquia).

La caracterización de la vegetación incluye trabajo en campo y en gabinete y sigue el protocolo mencionado en Rangel y Velázquez (1997). En el campo el procedimiento básico sirve para obtener la información sobre composición florística y aspectos de la estructura (cobertura, densidad, volumen, área basal). Las actividades analíticas incluyen la estimación de índices como el IVI y el IPF (Cantillo \& Rangel, 2011). En los últimos años se han utilizado aproximaciones metodológicas automatizadas como PC-ORD ${ }^{\circledR}$ para Windows versión 4.41 (McCune y Mefford, 1999) para segregar tendencias según la composición florística. Los grupos florísticos son definidos según las especies características dominantes y diferenciales y se describen siguiendo las recomendaciones del código de nomenclatura fitosociológica versión en español (Izco y Del Arco, 2003). Detalles del procedimiento metodológico se encuentran en Cleef et al. (1984), Rangel (2012b), Avella y Rangel (2012). 
Para el componente clima, los registros brutos de las estaciones pluviométricas y climatológicas representativas existentes en cada una de las regiones naturales fueron cedidos de manera gratuita o se adquirieron por compra directa en el Instituto de Hidrología, Meteorología y Estudios Ambientales (IDEAM). Los análisis esencialmente se enfocaron en la precipitación. Los tipos de clima se estimaron según la propuesta de Thornthwaite. Después de analizar los valores de precipitación, según las estaciones meteorológicas incluidas en el estudio de cada región y de acuerdo con el procedimiento metodológico que se sigue en nuestro grupo de investigación, se determinaron los valores mínimo y máximo del parámetro para establecer los límites que definieron las diferentes unidades que se mencionan en las caracterizaciones climáticas regionales (Caribe y Orinoquia).

Los parámetros básicos que se utilizan en la caracterización y en la definición de los ecosistemas se relacionan con las variaciones en la fisiografía, que diferencian claramente las planicies, de las colinas, las sierras, las serranías y los macizos, patrón que se repite en todas nuestras regiones naturales. En el mismo sentido, las grandes clases de clima permiten considerar en cualquiera de nuestras regiones naturales unidades asociadas con variaciones en los montos anuales. Los patrones en la precipitación se manifiestan de forma muy clara en la disposición de las diferentes fitocenosis, cuya composición florística y sus arreglos en la estructura, condicionan el establecimiento de la biota asociada. Cuando se unen la topografía y las variaciones del clima (monto anual de precipitación, valores medios, balance hídrico) se pueden definir unidades homogéneas de terreno.

En la parte biótica, el componente principal de nuestra aproximación metodológica para definir los ecosistemas, son los tipos de vegetación en un arreglo jerárquico, que guarda estrecha similitud con los enunciados teóricos de Mann (1964). La alianza, una de las unidades básicas del sistema fitosociológico, tiene composición característica de especies, ocupa un espacio vital definido (corología), presenta equilibrio poblacional dinámico (relaciones de abundancia, dominancia), sus componentes tienen independencia energética y muestra permanencia en el tiempo. En el componente animal, las aproximaciones se hacen sobre los patrones de riqueza de mamíferos, reptiles, aves y anfibios. Esta definición y alcance del término ecosistema encaja de buena manera con las "propiedades emergentes" como la vegetación o cobertura vegetal que se relaciona con el hábitat y su complejidad, así como con las geoformas que incluyen relieve, geología, geomorfología y características del suelo. Con estas variables se pueden "identificar y delimitar unidades homogéneas" (Etter, 1997). La definición de ecosistemas según esta aproximación metodológica ha sido ampliamente utilizada en Colombia en los últimos 30 años (Van der Hammen, 1984; 2003; 2005; Rangel-Ch. (2004b; 2012c).

\section{Resultados}

\section{Chocó biogeográfico (Costa Pacífica)}

Riqueza de las plantas con flores: En el componente vegetal existen registros de 4525 especies de plantas con flores que significan el $17 \%$ de la riqueza del país, 1211 géneros y 170 familias, de las cuales las más ricas son: Rubiaceae (72 géneros / 342 especies), Orchidaceae (94/250), Melastomataceae (27/225), Piperaceae (4/180), Araceae $(17 / 169)$ y Asteraceae (72/125). Entre las particularidades del patrón de riqueza cabe mencionar los valores bajos en Poaceae y los valores altos que muestran las familias Bromeliaceae, Clusiaceae, Gesneriaceae y Ericaceae. Los géneros con mayor número de especies son Piper (120), Psychotria (94), Anthurium (80), Miconia (72) y Clusia (54). El patrón de riqueza que se diferenció (Tabla 1) muestra que en las 15 familias más ricas del Chocó biogeográfico está representado el $49 \%$ de la riqueza total; cuando se incluyen a las 30 familias más ricas se tiene el $69 \%$ de esta riqueza. El resto de las familias (140) con 1419 especies representan el $31 \%$ de la riqueza.

A nivel genérico, los 15 géneros más ricos en especies significan el $18 \%$ de la riqueza total, valor que se incrementa hasta el 26\% cuando se consideran los 30 géneros más ricos. El resto de géneros (1181) representa la mayor concentración de la riqueza de especies (74\%).

\section{Riqueza de otros grupos de la flora y de la fauna (Rangel-} Ch., ed., 2004a)

En helechos se encontraron registros de 425 especies que significan el $28 \%$ de la riqueza total del grupo en Colombia. Otro grupo importante es el de las hepáticas con 170 especies, $20 \%$ de la riqueza del país. Los grupos con valores más bajos fueron los líquenes con 189 especies, 12\% de la riqueza a nivel nacional y los musgos con 132 especies, $14 \%$ de la riqueza del país. En la fauna, el grupo con mayor riqueza es el de las aves, 778 especies que representan el $42 \%$ de la riqueza del país, le siguen los mamíferos 180 especies, $37 \%$ y los reptiles 188 especies, $37 \%$. Los anfibios presentaron el valor más bajo con 137 especies, es decir $20 \%$ de la riqueza del país.

\section{Tipos de vegetación - Ecosistemas.}

En la caracterización de la vegetación del Chocó biogeográfico, Rangel (2004b) mencionó 86 tipos de vegetación o comunidades definidas según su composición florística y aspectos de la estructura, las cuales constituyeron la base para la definición y caracterización de 20 ecosistemas.

En la serie hídrica, los grandes ambientes se inician con los estuarios donde se establecen diferentes tipos de manglares dominados por Rhizophora mangle, por Rhizophora harrisonii, por Avicennia germinans, por Laguncularia racemosa y combinaciones especiales con Pelliciera rhizophorae. También aparecen los natales de Mora 
Tabla 1. Familias y géneros más ricos en el Chocó biogeográfico de Colombia.

\begin{tabular}{|c|c|c|c|}
\hline Familia & Géneros/especies & Géneros & Especies \\
\hline Rubiaceae & $72 / 342$ & Piper & 120 \\
\hline Orchidaceae & $94 / 250$ & Psychotria & 94 \\
\hline Melastomataceae & $27 / 225$ & Anthurium & 80 \\
\hline Piperaceae & $4 / 180$ & Miconia & 72 \\
\hline Araceae & $17 / 169$ & Clusia & 54 \\
\hline Asteraceae & $72 / 125$ & Peperomia & 53 \\
\hline Bromeliaceae & $3 / 119$ & Solanum & 45 \\
\hline Clusiaceae & $15 / 114$ & Cavendishia & 43 \\
\hline Fabaceae & $40 / 114$ & Ficus & 39 \\
\hline Gesneriaceae & $18 / 108$ & Heliconia & 38 \\
\hline Poaceae & $52 / 103$ & Passiflora & 38 \\
\hline Euphorbiaceae & $38 / 100$ & Calathea & 35 \\
\hline Ericaceae & $11 / 90$ & Columnea & 35 \\
\hline Cyperaceae & $20 / 82$ & Guzmania & 34 \\
\hline Arecaceae & $30 / 82$ & Philodendron & 33 \\
\hline Suma 15 familias más ricas & $2203(49 \%)$ & Suma 15 géneros más ricos & $813(18 \%)$ \\
\hline Otras 15 familias desde Solanaceae (81) hasta Verbenaceae (44) & $905(20 \%)$ & Otros 15 géneros (Pitcairnia hasta Aegiphila) & $362(8 \%)$ \\
\hline Suma 30 familias más ricas & $3108(69 \%)$ & Suma 30 géneros más ricos & $1175(26 \%)$ \\
\hline Resto de familias & $1419(31 \%)$ & Resto de géneros & $3352(74 \%)$ \\
\hline Total & 4527 & Total & 4527 \\
\hline
\end{tabular}

megistosperma (nato) y Rhizophora mangle. En total en los estuarios se registraron once (11) tipos de vegetación y se definieron dos ecosistemas.

En el ambiente fluviolacustre, se mencionaron a representantes de la vegetación acuática, de pantano y de la ribera (17 tipos), que incluyen desde los tapetes flotantes de Eichhornia crassipes y Pistia stratiotes hasta los palmares de Raphia taedigera (Zuluaga, 1987), que permitieron diferenciar tres ecosistemas.

En el ambiente de la llanura aluvial (21 tipos de vegetación) se establecen los bosques de Prioria copaifera (cativo), de Symphonia globulifera (machare) y varios tipos de guandales como el tangarial o bosques de Carapa guianensis y el sajal o bosques de Campnosperma panamense en la parte media de la región. En el Sur predominan varios tipos de guandales como los cuangariales dominados por especies de Virola y los naidizales dominados por Euterpe cuatrecasana; estos tipos de vegetación constituyeron la base para diferenciar cinco ecosistemas.

En las colinas bajas (20 tipos de vegetación) se establecen entre otros, los bosques de Huberodendron patinoi (carrá), de Dacryodes occidentalis y Otoba gracilipes y los palmares de Wettinia quinaria. En este ambiente se caracterizaron seis ecosistemas. En las colinas medias (once tipos de vegetación) dominan los bosques de Cavanillesia platanifolia y los palmares mixtos de Oenocarpus bataua y Cedrela odorata, que se asociaron con dos ecosistemas. En las colinas altas (seis tipos de vegetación) son frecuentes los bosques de Pseudolmedia laevigata, los de Anacardium excelsum y varias combinaciones de Brosimum utile que sirvieron para caracterizar dos ecosistemas.

La mayor diversidad en los tipos de vegetación se encontró en la llanura aluvial y en las colinas bajas.

\section{Patrones latitudinales y altitudinales en la distribución de la precipitación y de la biodiversidad (riqueza).}

De acuerdo con la distribución de los montos anuales de precipitación, Rangel y Arellano (2004) propusieron tres subregiones o zonas que guardan similitud con las propuestas de Trojer (1958). La zona en la cual se recibe más lluvia en todas sus unidades ecogeográficas, desde los estuarios hasta las colinas medias y altas es el centro $\left(<6^{\circ} 30^{\prime}-3^{\circ} 30^{\prime}\right)$, le sigue el Sur $\left(<3^{\circ} 30^{\prime}-1^{\circ}\right)$ y por último la porción Norte $\left(9^{\circ}-6^{\circ} 30^{\prime}\right)$ que incluye la zona del Darién con los valores más bajos. Al comparar el patrón latitudinal en la distribución de la lluvia con la riqueza (número total de especies) y la distintividad biológica (número de especies restringidas) en las plantas con flores y en los reptiles, se encuentra que los valores en la región central son mayores que en las otras dos franjas latitudinales. 
A nivel altitudinal, las unidades ecogeográficas localizadas entre el nivel del mar y $150 \mathrm{~m}$ o inclusive hasta $500 \mathrm{~m}$, son las que reciben mayor cantidad de lluvia, particularmente la llanura aluvial y las terrazas de la zona centro, donde se encuentran algunas de las localidades más lluviosas del mundo (Lloró). La mayor expresión de la riqueza se presenta en la llanura aluvial y en las terrazas.

En general, este patrón se podría interpretar como discriminante entre lo que son las tierras bajas que tipifican la denominación de Chocó biogeográfico y las estribaciones cordilleranas adyacentes. La marcha de la precipitación y la segregación latitudinal y topográfica es un argumento adicional, válido para confirmar la diferenciación del Chocó biogeográfico como una región natural en Colombia.

\section{Caribe}

Riqueza de las plantas con flores: Se tienen registros sobre 4274 especies de plantas con flores (Rivera y Rangel, 2012) de 1428 géneros y 203 familias, de las cuales las más diversificadas son Poaceae (113 géneros / 306 especies), Asteraceae (132/302), Fabaceae (61/196), Rubiaceae (59/175), Euphorbiaceae (43/141), Melastomataceae (24/106) y Orchidaceae (46/108). Entre las particularidades del patrón de riqueza cabe mencionar los valores altos en Poaceae y Asteraceae, probablemente por la inclusión de los macizos montañosos de la Serranía de Perijá y de la Sierra Nevada de Santa Marta en el patrón general de la región Caribe. Los géneros con mayor número de especies son Solanum (51), Miconia (50), Peperomia (38), Piper (36), Ficus (36), Psychotria (35) y Tillandsia (35). El patrón de riqueza que se detectó (Tabla 2) muestra que en las 15 familias más ricas del Caribe está representado el $47 \%$ de la riqueza total, cuando se aumenta a las 30 familias más ricas se tiene el $66 \%$ de esta riqueza. El resto de las familias (173) con 1430 especies representan el $34 \%$ de la riqueza. A nivel genérico, los 15 géneros más ricos en especies significan el $11 \%$ de la riqueza total. El resto de géneros (1413) representa la mayor concentración de la riqueza de especies $(89 \%)$.

\section{Riqueza de otros grupos de la flora y de la fauna (Rangel- Ch., ed., 2012a)}

En helechos se encontraron registros de 386 especies que significa el $25 \%$ en comparación con la riqueza total del grupo en Colombia. En hepáticas, especialmente en lo relativo a los macizos montañosos se poseen registros de 182 especies, $22 \%$ de la riqueza del país. En musgos hay registros de 230 especies, $24 \%$ de la riqueza del país y en líquenes de 379 especies, $24 \%$ de la riqueza del país. En fauna, el grupo con mayor riqueza es el de las aves con 917 especies que representan el 50\% de la riqueza del país, probablemente por la variedad de hábitats, donde figuran ambientes marinos, de estuario, ciénagas y pantanos y la variedad de ambientes en las planicies y en los macizos. Le siguen los mamíferos con 188 especies, $38 \%$ de la riqueza del grupo en Colombia y los reptiles con 185 especies
(36\%) de la riqueza del país. El grupo más pobremente representado es el de los anfibios con 104 especies, 15\% de la riqueza del grupo en el país.

\section{Tipos de vegetación - Ecosistemas}

La caracterización de la vegetación del Caribe se efectuó con base en la composición florística y en aspectos de la estructura (Dugand, 1970; Rieger, 1976; Lozano, 1984). Rangel (2012c) consideró criterios primarios como la fisiografía para diferenciar entre las tierras planas y las colinas, sierras y serranías a lo cual asoció la variación de los montos de precipitación según unidades climáticas, desde las que presentan montos de lluvia anuales menores a $600 \mathrm{~mm}$ hasta las más ricas o pluviales con montos superiores a los 3000 $\mathrm{mm}$ anuales (Rangel y Carvajal, 2012). En esa clasificación general, se relacionaron la serie topográfica o altitudinal y la serie pantanosa o de planicies. En esta última, los diversos ensambles vegetales incluyen desde bosques de manglar, bosques de pantano, matorrales, bosques espinosos, bosques secos con follaje caedizo o siempre verde, bosques ribereños, bosque tropical seco siempre verde, bosque húmedo hasta los bosques muy húmedos pluviales. En la serie altitudinal o topográfica las unidades de vegetación caracterizadas se adscribieron a las regiones tropical, subandina y andina y paramuna (Cuatrecasas 1958; Rangel et al., 1982).

En total se caracterizaron 161 tipos de vegetación, los cuales fueron la base para definir los ecosistemas. Una síntesis del tema, incluye a:

Manglares: vegetación de estuario dominada por las especies de mangles. Según Sánchez et al. (1997) y Cortés y Rangel (2011) se diferencian dos grandes formaciones de acuerdo con la variación en la salinidad del agua.

Casi en contacto con el mar, predominan los manglares de la gran formación o alianza fitosociológica, Avicennio germinantis- Rhizophorion manglis con las asociaciones Rhizophoretum manglis; Pelliciero rhizophorae Rhizophoretum manglis y Lagunculario racemosaeConocarpodetum erectae. Cuando disminuye la salinidad y hay mayor influencia del agua dulce, se establece la alianza Fico dendrocidae - Rhizophoro manglis con la asociación Priorio copaiferae-Rhizophoretum manglis (Cortés y Rangel, 2011; 2012).

Matorrales y herbazales semidesérticos: vegetación de porte bajo con un estrato arbustivo hasta de $1 \mathrm{~m}$ con elementos esparcidos ralos, follaje caedizo. Las especies dominantes son Lycium tweedianum, Heterostachys ritteriana, Sesuvium edmonstonei y Prosopis juliflora. La gran formación dominante o alianza fitosociológica es Lycio tweedianae - Sesuvion edmonstonei.

A nivel regional, cuando la precipitación aumenta, se pueden establecer:

Matorrales espinosos: vegetación de porte bajo con copas abiertas, donde los elementos armados son muy frecuentes. 
Tabla 2. Familias y géneros de las plantas con flores más ricos en la región Caribe de Colombia.

\begin{tabular}{|c|c|}
\hline Familia & Géneros/especies \\
\hline Poaceae & $113 / 306$ \\
\hline Asteraceae & $132 / 302$ \\
\hline Fabaceae & $61 / 196$ \\
\hline Rubiaceae & $59 / 175$ \\
\hline Euphorbiaceae & $43 / 141$ \\
\hline Melastomataceae & $24 / 106$ \\
\hline Orchidaceae & $46 / 108$ \\
\hline Mimosaceae & $25 / 104$ \\
\hline Solanaceae & $18 / 95$ \\
\hline Bromeliaceae & $11 / 77$ \\
\hline Cyperaceae & $18 / 82$ \\
\hline Piperaceae & $13 / 74$ \\
\hline Caesalpiniaceae & $20 / 70$ \\
\hline Acanthaceae & $20 / 71$ \\
\hline Bignoniaceae & $31 / 70$ \\
\hline Suma 15 familias más ricas & $1977(46 \%)$ \\
\hline Otras 15 familias desde Apocynaceae (70) hasta Cucurbitaceae (38) & $793(19 \%)$ \\
\hline Suma 30 familias más ricas & $2770(65 \%)$ \\
\hline Resto de familias (173) & $1430(33 \%)$ \\
\hline Total (203 familias) & 4274 \\
\hline
\end{tabular}

\begin{tabular}{|c|c|}
\hline Géneros & Especies \\
\hline Solanum & 51 \\
\hline Miconia & 50 \\
\hline Peperomia & 38 \\
\hline Piper & 36 \\
\hline Ficus & 36 \\
\hline Psychotria & 35 \\
\hline Tillandsia & 35 \\
\hline Pasiflora & 29 \\
\hline Ipomea & 28 \\
\hline Cyperus & 25 \\
\hline Croton & 25 \\
\hline Senna & 24 \\
\hline Paspalum & 23 \\
\hline Euphorbia & 23 \\
\hline Cordia & 21 \\
\hline Suma 15 géneros & $479(11 \%)$ \\
\hline Resto de géneros (1413) & $3721(87 \%)$ \\
\hline Total (1428 géneros) & 4274 \\
\hline
\end{tabular}

Las especies dominantes son Opuntia caracassana, Castela erecta, Caesalpinia coriaria, Malpighia punicifolia, Pereskia guamacho, Prosopis juliflora, Varronia globosa y Handroanthus billbergii (La Guajira). La gran formación dominante (clase fitosociológica) es Opuntio caracassanae Prosopietea juliflorae que incluye dos formaciones a nivel de orden fitosociológico Malpighio punicifoliae - Prosopietalia juliflorae (bosques ralos, bosques densos y bosques espinosos) y Parkinsonio praecocix - Casteletalia erectae (bosques achaparrados, bosques ralos, matorrales espinosos).

Cardonales: vegetación dominada por especies de cactaceas arborescentes con presencia ocasional de elementos arbóreos esparcidos. Las especies dominantes son Stenocereus griseus (cardón, yotojoro), Pilosocereus lanuginosus y Pereskia guamacho. La gran formación dominante a nivel de orden fitosociológico es Stenocereo grisei - Pereskietalia guamachae, representada en localidades de La Guajira, Magdalena y Atlántico.

Bosques secos estacionales con follaje caedizo: formación vegetal con amplia distribución en el Caribe. Tiene estratos arbóreos dominados por Handroanthus billbergii, Gyrocarpus americanus, Bursera graveolens, Coursetia caribaea y Pseudobombax septenatum. La formación dominante a nivel de alianza fitosociológica es Platymiscio pinnatae - Gyrocarpion americanae.
Bosques secos incluyendo vegetación ribereña de planicie: agrupa bosques que se establecen cerca de los cauces de ríos y quebradas, con elementos hasta $25 \mathrm{~m}$ de altura. Entre las especies dominantes figuran Anacardium excelsum, Hura crepitans, Aspidosperma polyneuron, Toxicodendron striatum, Enterolobium cyclocarpum y Astronium graveolens (Córdoba, Cesar, Magdalena). La formación dominante a nivel de orden fitosociológico es Huro crepitantis - Anacardietalia excelsi, que incluye varias formaciones boscosas dominadas por Anacardium excelsum (caracolí) como en el Parque Nacional Natural Tayrona.

Bosques húmedos: se desarrollan sobre colinas y terrazas bajas, en ocasiones en los alrededores de las ciénagas. Las especies dominantes son Matayba elegans, Pseudolmedia laevigata, Dialium guianense, Cavanillesia platanifolia, Tapirira guianensis y Protium heptaphyllum (Cesar, Córdoba, Bolívar). Entre las formaciones dominantes a nivel de alianza fitosociológica figuran los bosques del Dialio guianensis - Mataybion elegantis y los palmares mixtos de Astrocaryum malybo y Cavanillesia platanifolia.

Bosques húmedos - muy húmedos: vegetación boscosa y selvática desarrollada sobre sitios planos e inclinados. Entre las especies dominantes figuran Dendrobangia boliviana, Iryanthera hostmannii, Pentaclethra macroloba, Pseudolmedia laevigata y Naucleopsis glabra (Córdoba). 
A nivel de clase fitosociológica, domina la gran formación de Pentaclethra macroloba - Iryantheretea hostmannii que incluye a los bosques de la alianza Brosimo utilis Pentaclethrion macrolobae.

Bosques superhúmedos-pluviales (Córdoba): bosques y selvas que se desarrollan sobre terrenos estacionalmente inundables. Entre las especies dominantes figuran Peltogyne purpurea, Macrolobium ischnocalyx, Eschweilera coriacea, Dipteryx oleifera y Pentaclethra macroloba. A nivel de alianza fitosociológica domina la gran formación Eschweilero coriaceae - Pentachletrion macrolobae que incluye a los bosques dominados por Prestoea decurrens y Trichilia poeppigii y a los bosques de Macrolobium ischnocalyx y Peltogyne purpurea.

En la serie topográfica o altitudinal, se incluyen los tipos de vegetación caracterizados en la Sierra Nevada de Santa Marta, flanco Norte y en la Serranía de Perijá desde la parte baja o región tropical hasta la parte alta o región del páramo.

Sierra Nevada de Santa Marta, costado norte, filo Buritaca (Cleef et al., 1984; Pinto y Rangel, 2010a)

Región tropical $(<\mathbf{5 0 0}-\mathbf{1 1 0 0} \mathbf{m})$ : Gran formación de los bosques y selvas de la alianza fitosociológica Zygio longifoliae - Virolion sebiferae, donde se agrupan el bosque palmar del Zygio longifoliae-Dictyocaryetum lamarckianum y el bosque del Poulsenio armatae - Perseetum americanae.

Región subandina (>1100 - $\mathbf{2 4 0 0}$ m): Formación de los bosques de la alianza fitosociológica Gustavio speciosae - Tovomition weddellianae, que incluye al bosque palmar del Calatolo costaricensis - Dictyocaryetum lamarckiani y a los bosques del Cavendishio callistae - Tovomitetum weddellianae.

Región andina (>2400 - $\mathbf{3 1 0 0}$ m): Formación de los bosques achaparrados, raquíticos y bosques altos de la alianza fitosociológica Myrciantho ternifoliae - Weinmannion pinnatae que incluye a los bosques raquíticos del Chaetolepido santamartensis - Myrcianthetum ternifoliae y a los bosques altos del Clusio multiflorae - Weinmannietum pinnatae.

Región del páramo, incluye también el costado Sur, vertiente orientada hacia Valledupar $(>3100 \mathrm{~m})$

Matorrales de la alianza fitosociológica del Obtegomerio caerulescentis - Lachemillion polylepidis, donde se agrupan a los herbazales-rosetales del Valeriano karstenii Libanothamnetum occulti y a los matorrales pastizales del Arcytophyllo nitidi - Festucetum sanctae-martae.

Herbazales de la alianza fitosociológica Ranunculo spaniophylli - Calamagrostion effusae, que incluye a los rosetales-pajonales del Drabo cheiranthoidis Calamagrostietum effusae y al pastizal del Perissocoeleo purdiei - Calamagrostietum effusae.
Serranía de Perijá (Rangel y Arellano, 2009; Pinto y Rangel, 2010a)

Región tropical ( $<\mathbf{5 0 0}-\mathbf{1 2 0 0} \mathbf{~ m})$ : En las zonas Norte y centro de la Serranía, se establece la gran formación de las selvas, bosques altos y bosques ralos de la alianza fitosociológica Billio roseae - Maurion suaveolentis, que incluye a las formaciones boscosas del Caseario argutae - Aspidospermetum polyneurontis y al Neeo obovatae-Acalyphetum diversifoliae.

En la zona Sur (Aguachica, San Martín) se establecen los bosques de Astronium graveolens y Cavanillesia platanifolia y los bosques de Anacardium excelsum con especies de Inga y Ocotea (Avella \& Rangel, 2012)

Región subandina (>1200 - 2400 m): Gran formación boscosa de la alianza fitosociológica del Acalypho macrostachyae - Prunion intregrifoliae que incluye a los bosques del Piperi amalaginis -Lozanelletum enantiophyllae y a los bosques del Piperi lanceaefolium - Ingetum edulis. También se presentan en esta región los bosques de la asociación Styracis cordati - Alchorneetum grandiflorae. En la zona Sur (Aguachica, San Martín) se establecen los bosques de Heliocarpus americanus y los de Nectandra membranacea y Parathesis adenanthera.

Región andina (>2400 - $\mathbf{3 1 0 0}$ m): Gran formación de los bosques del Ilici sessiliflorae - Hesperomelion ferrugineae que reúne a la formación de bosques achaparrados del Paragynoxio martingrantii - Hesperomeletum ferrugineae y a los bosques altos del Clusio multiflorae - Weinmannietum pinnatae. También se encuentran en esta región los bosques de la asociación Merianio grandidentis - Styloceretum laurifoli.

\section{Región del páramo (>3100 m)}

Matorrales-pajonales de la alianza fitosociológica Hyperico baccharoidis - Calamagrostion effusae que incluye a los matorrales-pajonales del Bejario nanae - Calamagrostietum effusae y a los matorrales del Bejario resinosae Hypericetum magdalenici y del Gaylussacio buxifoliae Arcytophylletum nitidi.

Herbazales-pajonales de la alianza fitosociológica Lourteigio stoechadifoliae - Calamagrostion effusae que incluye a los pajonales frailejonales del Calamagrostio effusae Espeletietum perijaensis y a los pajonales-herbazales del Calamagrostio intermediae - Lourteigietum stoechadifoliae y del Calamagrostio effusae-Orthrosanthetum chimboracensis.

También se encuentran los chuscales de la alianza fitosociológica Hyperico stricti-Chusqueion tessellatae con los frailejonales-chuscales del Espeletio perijaensis Chusqueetum tessellatae, los chuscales del Geranio holosericei - Chusqueetum tessellatae y los matorrales-pajonales del Arcytophyllo nitidi - Calamagrostietum intermediae.

\section{Ecosistemas}

De acuerdo con Rangel (2012c y en prep.) se diferenciaron 54 ecosistemas, cuya distribución se inicia con los de 
estuario (3), los de ambientes fluvio-lacustres (4) y de la llanura aluvial (10) y se continúa con los ecosistemas de los matorrales áridos (5), de los cardonales, de los bosques secos-espinosos y de los boques secos estacionales (5), en localidades con valores bajos y medios de precipitación. La serie hídrica en estas planicies concluye con los ecosistemas de áreas con valores altos de precipitación, zonas húmedas, muy húmedas y pluviales (10). En las colinas y cerros bajos $(<500$ $\mathrm{m}$ de altitud) se diferenciaron a seis ecosistemas, mientras que en los macizos (Serranía de Perijá y Sierra Nevada de Santa Marta) se caracterizaron a 17 que incluyen a los ecosistemas boscosos de la región tropical hasta los ecosistemas de pajonales, frailejonales y herbazales del páramo.

\section{Patrones latitudinales en la distribución de la riqueza}

Rangel y Carvajal (2012) diferenciaron 14 unidades climáticas según el monto anual de la precipitación y el patrón de distribución de las lluvias, desde la unidad A con montos de lluvia menores a $600 \mathrm{~mm}$ anuales hasta la unidad $\mathrm{G}$ con montos superiores a los $3000 \mathrm{~mm}$ anuales. En la Tabla 3 se presenta la variación de la precipitación y su relación con la fisionomía y los tipos de vegetación en las planicies del Caribe, que ilustra de manera clara la gradación latitudinal desde los herbazales desérticos en localidades con montos de lluvia $<600 \mathrm{~mm}$, especialmente en La Guajira, al Norte la región Caribe y los bosques superhúmedos pluviales al Sur de Córdoba en localidades con montos de lluvia superiores a los $3000 \mathrm{~mm}$ anuales.

\section{Patrones altitudinales}

Para las variaciones altitudinales (topográficas), el estudio de caso más representativo lo muestra la Serranía de Perijá. En cada una de las regiones de vida, se presentan localidades con diferentes montos de precipitación (unidades climáticas) y tipo de clima según Thornthwaite. La mayor variabilidad se presenta en la región tropical. En la región de vida tropical (0 - $1000 \mathrm{~m}$ ), el monto anual de lluvia varía entre $894 \mathrm{~mm}$, en el sector Norte con tipos de clima según Thornthwaite DS2A' hasta $2500 \mathrm{~mm}$ en el Sur sectores alrededor de San Alberto y La Gloria con tipo de clima según Thornthwaite C2W'2A'. Los regímenes de distribución de lluvia son bimodalestetraestacionales; las épocas lluviosas se presentan entre abril y junio y entre agosto y noviembre.

En la región subandina (1000 a $2000 \mathrm{~m}$ de altitud), los montos anuales de precipitación están por encima de $1400 \mathrm{~mm}$. Hay muy pocas estaciones meteorológicas en esta región. De acuerdo con Rangel y Arellano (2009) se establecen bosques nublados, húmedos y muy húmedos de la alianza fitosociológica Guatterio columbianaePseudolmedion rigidae, que incluye palmares mixtos y bosques altos pluriestratificados que se relacionan con valores altos de precipitación (>1400 mm), buena capacidad de almacenamiento y regulación de agua en los suelos.

En la alta montaña de Perijá, regiones andina y páramo $(>2500$ a $3500 \mathrm{~m}$ ), las localidades con valores de precipitación entre 1000 y 1400 mm están representadas en el sector Norte y las que presentan los valores más altos $(>1800 \mathrm{~mm})$ se localizan en el Sur. En general, el patrón de segregación latitudinal de los montos de lluvia, califica a la parte Sur como la más húmeda, el Norte con los valores menores y el centro con valores intermedios.

El inventario de la riqueza en grupos de la flora y de la fauna (varios autores en Rangel-Ch., ed., 2009), muestra la siguiente situación; se encontraron registros de 1994 especies

Tabla 3. Variabilidad de la precipitación y su relación con el arreglo fisionómico y con la composición florística de la vegetación en el Caribe Colombiano.

\begin{tabular}{|c|c|c|c|c|c|}
\hline $\begin{array}{l}\text { Unidad climática } \\
(\mathrm{mm}) \text { anuales }\end{array}$ & Departamento & Región & $\begin{array}{l}\text { Período } \\
\text { de lluvias }\end{array}$ & Fisionomía & Tipo vegetación \\
\hline \multirow{2}{*}{$\begin{array}{l}\mathrm{A}^{\prime} \\
<600\end{array}$} & \multirow{2}{*}{ La Guajira } & \multirow{2}{*}{ U-bi } & \multirow{2}{*}{$\begin{array}{l}\text { Agosto- } \\
\text { noviembre }\end{array}$} & \multirow{2}{*}{ Herbazal desértico } & $\begin{array}{l}\text { Matorrales - herbazales postrados de Lycium tweedianum - } \\
\text { Sesuviun edmonstonei }\end{array}$ \\
\hline & & & & & $\begin{array}{l}\text { Bosques ralos - espinosos - cardonales de Opuntia } \\
\text { caracassana - Prosopis juliflora }\end{array}$ \\
\hline $\begin{array}{c}\text { A } \\
>600-1000\end{array}$ & Bolívar - Cesar & U-bi & $\begin{array}{l}\text { Mayo- } \\
\text { noviembre }\end{array}$ & Matorral espinoso & $\begin{array}{l}\text { Bosques espinosos - ralos de Stenocereus griseus - Pereskia } \\
\text { guamacho }\end{array}$ \\
\hline \multirow{2}{*}{$\begin{array}{c}\text { B } \\
1000-1400\end{array}$} & \multirow{2}{*}{$\begin{array}{l}\text { Magdalena - } \\
\text { Cesar - Sucre }\end{array}$} & \multirow{2}{*}{ B-te } & Abril-mayo & \multirow{2}{*}{$\begin{array}{l}\text { Bosques secos, } \\
\text { follaje caedizo }\end{array}$} & Bosque de Platymiscium pinnata y Gyrocarpus americana \\
\hline & & & Julio-octubre & & Bosque de Aspidosperma polyneuron - Anacardium excelsum \\
\hline $\begin{array}{c}\text { C } \\
1400-1800\end{array}$ & Córdoba - Cesar & U-bi & Abril-octubre & $\begin{array}{l}\text { Bosques secos- } \\
\text { semihúmedos }\end{array}$ & Bosque de Cavanillesia platanifolia - Astrocaryum malybo \\
\hline $\begin{array}{c}\text { D } \\
1800-2200\end{array}$ & Córdoba - Sucre & U-bi & $\begin{array}{l}\text { Abril- } \\
\text { noviembre }\end{array}$ & Bosques húmedos & Bosque de Dialium guianense - Matayba elegans \\
\hline $\begin{array}{c}\mathrm{E} \\
>2200-2600\end{array}$ & Córdoba & U-bi & $\begin{array}{c}\text { Mayo- } \\
\text { noviembre }\end{array}$ & $\begin{array}{l}\text { Bosques húmedos- } \\
\text { muy húmedos }\end{array}$ & Bosque de Brosimum utile - Pentacletha macroloba \\
\hline $\begin{array}{c}F \\
>2600-3000\end{array}$ & Córdoba & U-bi & $\begin{array}{c}\text { Mayo- } \\
\text { noviembre }\end{array}$ & $\begin{array}{l}\text { Bosques húmedos- } \\
\text { superhúmedos }\end{array}$ & Bosque de Eschweilera coriacea - Pentacletha macroloba \\
\hline
\end{tabular}


de plantas con flores, de 246 de musgos y 182 de hepáticas. En reptiles hay registros de 71 especies, en mamíferos de 85 , en anfibios 44, en aves 391 y en mariposas 465 . En el gradiente altitudinal, la región subandina presentó la mayor expresión de riqueza en todos los grupos, a saber: 942 especies de plantas con flores, 150 de musgos y 111 de hepáticas. En fauna se encontraron 22 especies de reptiles, 68 de mamíferos, 19 de anfibios, 245 de aves y 80 de mariposas.

Los valores más bajos se encontraron en la región del páramo con 388 especies de plantas con flores, 91 de musgos y once de hepáticas. En fauna hay tres especies de reptiles, seis de mamíferos, cinco de anfibios, 86 de aves y 14 de mariposas.

\section{Orinoquia}

Riqueza de las plantas con flores: En el inventario de las plantas con flores se encontraron registros de 4347 especies (el 16.4\% de la riqueza del país) de 1260 géneros y 177 familias, de las cuales las familias con mayor número de géneros y especies fueron Rubiaceae (109 géneros / 683 especies), Poaceae (78/271), Fabaceae (58/216), Melastomataceae (37/207) y Asteraceae (81/144). Los linajes (géneros) con mayor número de especies fueron Psychotria (146), Miconia (71), Paspalum (51), Piper (44) y Palicourea (43).

El patrón de riqueza que se diferenció (Tabla 4) muestra que en las 15 familias más ricas está representada el 54\% de la riqueza total, cuando se aumenta a las 30 familias más ricas se tiene el $72 \%$ de esta riqueza. El resto de las familias (147) con 1215 especies representan el $27.9 \%$ de la riqueza. A nivel genérico, los 15 géneros más ricos en especies significan el $15 \%$ del total, cuando se aumenta a los 30 géneros más ricos se tiene el $22.4 \%$ de la riqueza. El resto de géneros (1230) representa la mayor concentración de la riqueza de especies (77.5\%).

\section{Riqueza de otros grupos de la flora y de la fauna}

Rincón et al. (2014) encontraron registros de 86 especies de musgos ( $8 \%$ con relación a la riqueza de Colombia), 130 de líquenes (8\%) y 254 de helechos (17\%). En fauna (Rangel. ed. 2014), hay una riqueza alta en aves con 761 especies que significa el $41.1 \%$ del total del país; en la llanura aluvial hay mayor diversidad (528 especies) alrededor de los caños, esteros y madreviejas (garzones, soldados, garzas, caracaras). En mamíferos, tanto terrestres como voladores, hay 196 especies (39.8\% del total del país). Los reptiles están representados por 128 especies ( $24 \%$ del país) y el grupo con menor número de especies es el de los anfibios (sapos y ranas), con 70 especies (10\% del país).

Tipos de vegetación - Ecosistemas (Rangel-Ch. \& Minorta-C., 2014; Rangel-Ch., 2014)

Vegetación: El inventario más reciente señala la presencia de 90 comunidades o formaciones vegetales con predominio de los bosques. Según las unidades fisiográficas, se presenta la siguiente condición:

Tabla 4. Familias y géneros de las plantas con flores más ricos en la Orinoquia de Colombia.

\begin{tabular}{|c|c|c|c|c|}
\hline Familia & Géneros & Especies & Géneros & Especies (\%) \\
\hline Rubiaceae & 109 & 683 & Psychotria & $146(3,5)$ \\
\hline Poaceae & 78 & 271 & Miconia & $71 \quad(1,6)$ \\
\hline Fabaceae & 58 & 216 & Paspalum & $51 \quad(1,7)$ \\
\hline Melastomataceae & 37 & 207 & Palicourea & $43 \quad(1,0)$ \\
\hline Asteraceae & 81 & 144 & Piper & $44 \quad(1,0)$ \\
\hline Cyperaceae & 21 & 131 & Inga & $39(0,9)$ \\
\hline Mimosaceae & 21 & 102 & Rhynchospora & $38 \quad(0,9)$ \\
\hline Euphorbiaceae & 38 & 99 & Solanum & $36 \quad(0,8)$ \\
\hline Orchidaceae & 48 & 92 & Faramea & $26(0,6)$ \\
\hline Caesalpiniaceae & 26 & 87 & Clusia & $31 \quad(0,7)$ \\
\hline Piperaceae & 2 & 70 & Rudgea & $30 \quad(0,7)$ \\
\hline Bignoniaceae & 23 & 68 & Passiflora & $28 \quad(0,6)$ \\
\hline Apocynaceae & 23 & 66 & Peperomia & $26(0,6)$ \\
\hline Araceae & 15 & 65 & Protium & $26(0,6)$ \\
\hline Solanaceae & 12 & 66 & Paullinia & $24(0,6)$ \\
\hline Suma 15 familias más ricas $(49,4 \%)$ & $592(47,0 \%)$ & $2367(54,0 \%)$ & Suma 15 géneros más ricos & $659(15,0)$ \\
\hline Suma 30 familias más ricas & $800(63,4 \%)$ & $3132(72,0 \%)$ & Suma 30 géneros más ricos & $974(22,4)$ \\
\hline Resto de familias (147) & $460(36,5 \%)$ & $1215(27,9 \%)$ & Resto de géneros (1230) & $3373(77,5)$ \\
\hline Total & 1260 & 4347 & Total & 4347 \\
\hline
\end{tabular}


Piedemonte: predominan los bosques (doce tipos), también se encuentran pastizales (cuatro); las formaciones con mayor área de distribución geográfica incluyen:

Bosque de Nectandra sp., Cecropia sp., Pseudolmedia laevigata y Chrysophyllum sp.

Bosques de Pourouma guianensis y Rinorea macrocarpa

Bosques de Cassia moschata

Palmares de Socratea exorrhiza e Iriartea deltoidea

Bosques-palmar de Oenocarpus bataua y Hieronyma alchorneoides

Bosque-palmar de Guarea guidonia, Cecropia membranacea y Socratea exorrhiza

Bosque de Calophyllum brasiliense, Enterolobium cyclocarpum y Garcinia floribunda

En la serranía La Macarena, las comunidades de mayor área de distribución incluyen a:

Bosques de Billia rosea y Brosimum utile

Bosques de Virola carinata y Chimarrhis turbinata

Bosques de Matayba guianensis y Virola elongata

Palmar mixto de Cespedesia spathulata y Euterpe precatoria

Bosques de Pourouma minor y Zanthoxylum flavum

Altillanura: se encontraron 18 tipos de bosque, tres matorrales, 16 pastizales y otros cuatro tipos. Entre los de mayor área de distribución geográfica figuran:

Pastizal-arbustivo (sabana) de Trachypogon spicatus y Axonopus purpusii

Palmar mixto de Oenocarpus bataua, Socratea durissima y Astrocaryum vulgare

Bosque de Schefflera morototonii y especies de Aspidosperma, Nectandra y Brosimum

Pastizal con Aristida riparia, Trachypogon spicatus, Rhynchospora bulbosa y Bulbostylis paradoxa

Bosque bajo de Campsiandra comosa (EL Tuparro)

Morichal-palmar de Mauritia flexuosa

Bosque de Jacaranda copaia, Qualea rosea y Parkia pendula (El Tuparro).

Rosetal herbáceo de Pitcairnia aff. mituensis, Chelonanthus sp. y Vellozia tubiflora (El Tuparro).

Bosque de Pseudolmedia laevigata, Virola elongata y Licania apetala

Llanura aluvial: se encontraron 15 tipos de bosque, 11 pastizales, un matorral y otros seis tipos. Entre los de mayor área de distribución figuran:
Bosque de Guarea sp., Crepidospermum rhoifolium, Pera arborea e Himatanthus articulatus

Bosque bajo de Cecropia peltata, Spondias mombin y Socratea durissima

Bosque alto de Crepidospermum rhoifolium, Pourouma guianensis y Pera arborea

Bosque de Nectandra sp., Manilkara bidentata y

Cecropia cf. peltata

Bosque alto de Pseudolmedia laevigata, Pouteria caimito y Luehea seemannii

Bosque de Caraipa guianensis y Caraipa llanorum

Bosques de Protium tenuifolium, Tabebuia serratifolia, Spondias mombin con Himatanthus articulatus, Socratea durissima y Astrocaryum chambira

Bosque de Laetia corymbulosa e Inga bonplandiana

Bosque bajo de Caraipa llanorum y especies de Aspidosperma y Pouteria

Pastizal de Paspalum carinatum, P. convexum e Ipomoea schomburgkii

Palmar de Acrocomia aculeata

Pastizal húmedo de Andropogon (A. selloanus; A. virgatus).

Pastizal-arbustivo de Trachypogon spicatus (ligularis) y Paspalum carinatum.

Pastizal de Trachypogon spicatus, Paspalum hyalinum y Mesosetum loliiforme.

Pastizal (sabana) de Anthaenantia lanata

En la llanura aluvial y en áreas especiales asociadas con zonas encharcadas o en las orillas de ríos y caños, se establecen los palmares mixtos con restos de bosque dominados por Mauritia flexuosa (moriche), que juegan un papel muy importante en el mantenimiento de la economía hídrica regional. También se pueden encontrar variados pastizales y herbazales.

En las depresiones del terreno "bajos" y en los esteros se establecen comunidades acuáticas y de pantanos que incluyen a los herbazales de Echinodorus tenellus, de Eleocharis minima, de Cabomba furcata (sumergido) y a los pastizales de Leersia hexandra, Paspalum repens (gramalote) y Ludwigia inclinata (clavito). En las orillas de las madreviejas flotan los tapetes de Eichhornia crassipes (taruya).

Finalmente en el extremo Oriental de la región, se presentan afloramientos del escudo guayanés (andén orinocense). Sus herbazales y rosetales están dominados entre otras especies, por Vellozia tubiflora y Pepinia pruinosa y presentan afinidad con los de los "tepuyes" o macizos guayaneses inmersos en la planicie amazónica. 


\section{Ecosistemas}

Se diferenciaron diez ecosistemas que tiene como esqueleto vegetal a diversos tipos de bosque (piedemonte); tres son palmares puros o mixtos (piedemonte, llanura aluvial); uno tiene como esqueleto vegetal a un matorral-bosque bajo (altillanura) y ocho tienen como esqueleto vegetal a pastizales (altillanura-llanura aluvial-eólica), a saber:

Ecosistema de los bosques de Swartzia macrophylla y Pseudolmedia laevigata, se establece en el piedemonte y en la llanura aluvial.

Ecosistema de los bosques de Pouteria caimito y Pseudolmedia laevigata, en la llanura aluvial.

Ecosistema de los bosques de Crepidospermum rhoifolium y Pera arborea, en la llanura aluvial y en la altillanura.

Ecosistema de los bosques de Faramea occidentalis y Terminalia amazonia, en la llanura aluvial

Ecosistema de los matorrales - bosques bajos dominados por Curatella americana y Byrsonima crassifolia, en la altillanura

Ecosistema de los palmares de Mauritia flexuosa, en la llanura aluvial y en la altillanura

Ecosistema de los bosques con Bocageopsis multiflora, Parkia pendula y Sclerolobium bracteosum, en la altillanura

Ecosistema de bosques de Billia rosea y Brosimum utile, en el piedemonte y en la altillanura

Ecosistema de los bosques de Cassia moschata, en el piedemonte

Ecosistema de los palmares con Socratea exorrhiza e Iriartea deltoidea, en el piedemonte

Ecosistema de los bosques de Batocarpus orinocensis y Senefeldera verticillata, en el piedemonte de la serranía La Macarena

Ecosistema de los bosques de Amaioua guianensis y Virola elongata, en el piedemonte de la serranía La Macarena

Ecosistema de los bosques de Laetia corymbulosa e Inga bonplandiana, en el piedemonte de la serranía La Macarena y en la llanura aluvial

Ecosistema del bosque - palmar de Guarea guidonia, Cecropia membranacea y Socratea exorrhiza, en la llanura aluvial

Ecosistema de los pastizales-herbazales de Trachypogon spicatus, Rhynchospora ciliata y Bulbostylis junciformis, en la altillanura y en la llanura aluvial

Ecosistema del pastizal de Trachypogon spicatus y Mesosetum lofiiforme, en la llanura aluvial
Ecosistema del pastizal de Andropogon leucostachyus y Otachyrium versicolor, en la altillanura

Ecosistema del pastizal en zanjas de zuros de Steinchisma (P.) laxa y Cyperus haspan, en la llanura aluvial

Ecosistema del pastizal en terraza baja de Aristida capillacea y Paspalum multicaule, en la llanura aluvial y en el piedemonte

Ecosistema del pastizal Anthaenantia (Leptocoryphium) lanata, Axonopus purpusii y Andropogon virgatus, en la llanura aluvial y en la altillanura

Ecosistema del pastizal de Paspalum carinatum, en la llanura aluvial, en la altillanura y en el piedemonte

Ecosistema del pastizal - matorral de Andropogon bicornis y Lippia alba, en la altillanura

La particularidad que exhibe la Orinoquia en cuanto al papel importante de los ecosistemas cuyo esqueleto vegetal son pastizales, es única para Colombia. Solamente en la alta montaña, en la región del páramo, se presentan igualmente numerosos ecosistemas cuyo esqueleto vegetal está dominado por las especies de pastos.

Aunque no se tiene información sobre la riqueza de los componentes de la biota referida a superficies plenamente delimitadas, los inventarios de las áreas geográficas en las cuales están representados, permiten diferenciar ecosistemas muy ricos en flora y en fauna asociada, como los de la Serranía de La Macarena (piedemonte) que incluyen al ecosistema del bosque - palmar de Guarea guidonia, Cecropia membranacea y Socratea exorrhiza con una riqueza de especies de plantas con flores entre 662-1000; 29 especies de reptiles, 24 de anfibios, 43 de mamíferos y 213 de aves. También figura, el ecosistema de la gran formación de los bosques de Batocarpus orinocensis y Senefeldera verticillata con cerca de 1134 especies de plantas con flores, 35 especies de reptiles, 30 de anfibios, 67 de mamíferos y 181 de aves. El ecosistema de los bosques de Billia rosea y Brosimum utile tiene 943 especies de plantas con flores, 25 especies de reptiles, 25 de anfibios, 35 de mamíferos y 181 de aves.

En la llanura aluvial en el ecosistema de los bosques de Pouteria caimito y Pseudolmedia laevigata se encontraron registros de 529 especies de plantas con flores, 14 especies de reptiles, 22 de anfibios, 49 de mamíferos y 44 de aves.

Los ecosistemas con esqueleto vegetal de tipo pastizal, en general fueron los que presentaron valores más bajos en los diferentes componentes de la biodiversidad, aunque el ecosistema del pastizal-herbazal de Trachypogon spicatus, Rhynchospora ciliata y Bulbostylis junciformis mostró valores parecidos a los de ecosistemas boscosos con 619 especies de plantas con flores, 29 especies de reptiles, $22 \mathrm{de}$ anfibios, 18 de mamíferos y 56 de aves. Igualmente figura el 
ecosistema del pastizal - matorral de Andropogon bicornis y Lippia alba con una riqueza de especies de plantas con flores entre 183-300, 35 especies de reptiles, 22 de anfibios, 25 de mamíferos y 60 de aves (Rangel, 2014).

\section{Distribución de la precipitación y de la biodiversidad (riqueza) en las unidades fisiográficas}

Según los montos anuales de precipitación, el piedemonte presenta los valores más altos (1637 - 4664), con tipos de clima según Thortnthwaite, superhúmedos y húmedos, seguido por la altillanura (1592 - 3100) con tipos de clima muy húmedos hasta medianamente húmedos. La casi totalidad de los regímenes de distribución de las lluvias son unimodales-biestacionales, aunque se presentan excepciones con regímenes bimodales-tetraestacionales, especialmente en áreas cercanas a mesas, mesetas o macizos.

El patrón de riqueza regional en las plantas con flores a nivel de familia que encabezan Rubiaceae, Poaceae, Fabaceae, Melastomataceae y Asteraceae, se repite en las unidades fisiográficas. El mayor número de especies se presenta en el piedemonte con 3150 especies y el más bajo en la llanura aluvial con 1131. Entre las particularidades que se detectaron, figura la mayor riqueza de Rubiaceae y Cyperaceae en la altillanura.

En grupos de fauna como reptiles (98 especies) y mamíferos (117 especies), en el piedemonte se presenta mayor riqueza, le sigue la altillanura. Entre las particularidades de la concentración de riqueza de fauna, figura el elevado número de especies de aves en la llanura aluvial (528 especies) y el de reptiles (101 especies) en la altillanura.

\section{Región cordillerana o andina (sin incluir al páramo)}

Los análisis se fundamentan en los resultados de transectos altitudinales desarrollados en los tres ramales andinos (ECOANDES, Van der Hammen, 1984) y en las investigaciones detalladas siguiendo la misma aproximación metodológica desarrolladas en el macizo central (P.N.N. Puracé - valle del Magdalena) y en localidades aledañas (Rangel y Lozano, 1986).

Riqueza de la flora y de la fauna: El número más probable de especies de plantas con flores se estima en 11500, que significa el $43 \%$ de la riqueza total del país. En la Tabla 5, aparecen las familias con mayor número de especies en los macizos montañosos con inventarios detallados a lo largo de su gradiente altitudinal.

Las familias más ricas son Asteraceae, Orchidaceae, Rubiaceae, Poaceae y Melastomataceae. En la cordillera central, transecto del Parque Nacional Natural Los Nevados, son importantes los valores de las familias Solanaceae y Lauraceae. En la c. Oriental - Transecto del Sumapaz, alcanzaron valores altos en la riqueza Clusiaceae y Rosaceae. En la c. Occidental - Transecto del Tatamá, las familias Araceae, Gesneriaceae, Melastomataceae, Bromeliaceae y
Ericaceae alcanzaron valores altos cuando se les compara con los de los otros macizos.

En helechos, se encontraron registros de 1050 especies, $69 \%$ de la riqueza total del grupo en Colombia. En musgos se cuenta con registros de 800 especies, es decir el $82 \%$ de la riqueza global; en líquenes $1300(83 \%)$ y en hepáticas $756(90 \%)$. En grupos de la fauna, se cuenta con registros de 329 especies de mamíferos (Solari et al., 2013, 71\% del país), en aves 974 especies (52\% de la riqueza del país), en anfibios (Roa y Ruiz, 1993; Ruiz et al., 1996; Acosta, 2000) 484 especies (69\% del país) y en reptiles 274 especies (54\% del país).

\section{Tipos de vegetación}

Tomando como base la documentación inicial de Rangel et al. (1997) y los trabajos detallados de ECOANDES en las tres cordilleras (Cleef et al., 2003; Rangel et al., 2005b, 2008) y contribuciones en otras áreas como el macizo central, transecto valle del Magdalena - volcán del Puracé (Rangel y Lozano 1986) y otras localidades de los Andes (Cantillo \& Rangel, 2011; Rangel y Avella, 2011; Avella y Rangel, 2014), se presentan los tipos de bosque caracterizados con base en el enfoque fitosociológico en los tres ramales cordilleranos de Colombia.

\section{Cordillera Occidental (Macizo del Tatamá)}

Los bosques achaparrados de la franja altoandina entre 3300 y $3700 \mathrm{~m}$ en las dos vertientes del macizo, se agrupan en el orden fitosociológico Myrsino dependentis-Escallonietalia myrtilloides que incluye la formación a nivel de alianza fitosociológica Desfontaineo parvifoliae-Ilicion supremae igualmente presente en las dos vertientes del macizo. La alianza agrupa a los bosques de Weinmannia engleriana y Miconia gleasoniana (Weinmannio englerianae-Miconietum gleasonianae) entre 3520 y $3700 \mathrm{~m}$ y a los bosques de Clusia clusioides y Clethra fagifolia (Clusio clusioidesClethretum fagifoliae) entre 3300 y $3500 \mathrm{~m}$. También se encuentran en la vertiente Occidental, los bosques de Schefflera ferruginea y Miconia latifolia (Schefflero ferrugineae y Miconietum latifoliae) entre 3300 y $3440 \mathrm{~m}$ y los bosques de Ocotea calophylla y Clusia multiflora a $2860 \mathrm{~m}$.

En la región andina entre 2500 y $3200 \mathrm{~m}$ en las dos vertientes, se establecen los bosques de la alianza fitosociológica de Geonoma weberbaueri y Drimys granadensis (Geonomo weberbauerii-Drimion granadensis) que incluye a los bosques de Hedyosmum bonplandianum y Prunus integrifolia (Hedyosmo bonplandiani-Prunetum integrifoliae) entre 2700 y $3130 \mathrm{~m}$ en las dos vertientes y a los de Weinmannia trianaea (Arthrostylidio venezuelae-Weinmannietum trianaea) entre 2910 y 3100 m en la vertiente Oriental. En la vertiente Oriental se encuentran los bosques de Quercus humboldtii (Pouterio lucumae-Quercetum hzumboldtii) entre 2100 y $2500 \mathrm{~m}$. 
Tabla 5. Familias de plantas con flores con mayor número de especies en macizos montañosos de la región Andina de Colombia

\begin{tabular}{|c|c|c|c|c|}
\hline Familia & $\begin{array}{c}\text { Cordillera Central Parque Nacional } \\
\text { Natural los Nevados }\end{array}$ & $\begin{array}{c}\text { Cordillera Oriental } \\
\text { Macizo Sumapaz }\end{array}$ & $\begin{array}{l}\text { Macizo Central } \\
\text { Puracé }\end{array}$ & $\begin{array}{l}\text { Cordillera Occidental } \\
\text { Macizo Tatamá }\end{array}$ \\
\hline Asteraceae & 161 & 190 & 75 & 106 \\
\hline Orchidaceae & 41 & 94 & 76 & 61 \\
\hline Rubiaceae & 76 & 63 & 38 & 90 \\
\hline Poaceae & 47 & 71 & 21 & 42 \\
\hline Melastomataceae & 52 & 75 & 40 & 99 \\
\hline Leguminosae & 55 & 46 & 5 & 52 \\
\hline Solanaceae & 53 & 38 & 38 & 37 \\
\hline Piperaceae & 55 & 30 & 40 & 49 \\
\hline Ericaceae & 27 & 37 & 57 & 62 \\
\hline Euphorbiaceae & 21 & 32 & 5 & 30 \\
\hline Araceae & 40 & 29 & 12 & 40 \\
\hline Lauraceae & 43 & 34 & 7 & 23 \\
\hline Bromeliaceae & 10 & 35 & 10 & 36 \\
\hline Clusiaceae & 20 & 38 & 5 & 37 \\
\hline Gesneriaceae & 6 & 12 & 6 & 60 \\
\hline Moraceae & 26 & 12 & 6 & 25 \\
\hline Rosaceae & 26 & 32 & 14 & 12 \\
\hline Suma de las 17 familias más ricas & $759(47,3 \%)$ & $868(57,8 \%)$ & $455(43,8 \%)$ & $861(54,3 \%)$ \\
\hline Resto de familias & $844(52,7 \%)$ & $633(42,2 \%)$ & $583(56,2 \%)$ & $725(45,7 \%)$ \\
\hline Total & 1603 & 1501 & 1038 & 1586 \\
\hline Área del macizo km2 & $110.000 *$ & $>130.000^{*}$ & ND & $76.000 *$ \\
\hline
\end{tabular}

* Superficie de toda la cordillera; no se tienen datos concretos sobre el área cubierta en los transectos.

En las regiones de vida subandina y tropical (2300 a 550 m) en las dos vertientes, se establece la vegetación de la gran formación a nivel de orden de los bosques dominados por Elaeagia utilis y Ossaea bracteata (Elaegio utilisOssaetalia bracteatae). Se diferencian las formaciones de palmares mixtos dominados por Calatola costaricensis y Wettinia kalbreyeri de la alianza fitosociológica Calatolo costaricensis-Wettinion kalbreyeri, entre 1350 y 2500 m en las dos vertientes, donde se reúnen a los bosques de Guettarda chiriquensis y Weinmannia balbisiana (Guettardo chiriquensis-Weinmannietum balbisianae) entre 1350 y 2500 $\mathrm{m}$ en la vertiente Occidental y a los palmares mixtos de Aiphanes simplex y Ocotea ira (Aiphano simplici-Ocoteetum irae) entre 1700 y $2150 \mathrm{~m}$ en la vertiente Oriental. La otra alianza fitosociológica es la de los bosques de Cecropia peltata y Brosimum utile (Cecropio peltatae-Brosimion utile) entre 550 y $1200 \mathrm{~m}$ en la vertiente Occidental, que incluye a los bosques de Jacaranda hesperia e Inga pavonia (Jacarando hesperiae e Ingetum pavoniae) entre 550 y 800 m; y a los bosques de Trichipteris procera y Nectandra reticulata (Trichiptero procerae-Nectandretum reticulatae) entre 800 y $1200 \mathrm{~m}$.
En la región tropical de la vertiente Oriental, se encuentran los bosques de Callicarpa acuminata y Ficus hartwegii (Callicarpo acuminatae-Ficetum hartwegii) entre $1150 \mathrm{y}$ 1700 en la vertiente Oriental.

\section{Cordillera Central (Parque Nacional Natural Los Nevados)}

Los bosques achaparrados de la franja altoandina entre 3250 y 3750 en la vertiente Occidental del macizo se agrupan en la alianza fitosociológica Neurolepido aristataeOreopanacion nitidi, que incluye a los bosques de Gordonia speciosa y Weinmannia pubescens (Gordonio speciosaeWeinmannietum pubescentes) entre 3250 y $3450 \mathrm{~m}$; a los bosques de Monochaetum lindenianum y Weinmannia mariquitae (Monochaeto lindeniani-Weinmannietum mariquitae) entre 3450 y $3650 \mathrm{~m}$ y a los bosques de Gynoxys baccharoides y Hesperomeles lanuginosa (GynoxyoHesperomeletum lanuginosae) entre 3550 y 3700 m.

En la vertiente Oriental, la vegetación entre 3300 y 3750 $\mathrm{m}$, se agrupa en la alianza fitosociológica Diplostephio bicoloris-Hesperomelion lanuginosae, donde se incluyen a los bosques de Hedyosmum bonplandianum (Chusqueo 
scandentis-Hedyosmetum bonplandiani) entre 3300y $3500 \mathrm{~m}$ y a los bosques bajos-matorrales de Gynoxys baccharoides y Diplostephium floribundum (Gynoxyo baccharoidesDiplostephietum floribundi) entre 3600 y $3750 \mathrm{~m}$. En la región andina entre 2800 y $3250 \mathrm{~m}$ se establecen los bosques de Weinmannia rollottii, alianza fitosociológica (Chusqueo scandentis-Weinmannion rollottii), que incluye a los bosques de Tovomita guianensis y Clusia multiflora (Tovomito guianensis-Clusietum multiflorae) entre $2800 \mathrm{y}$ $3050 \mathrm{~m}$ y a los de Brunellia goudotii (Chusqueo scandentisBrunellietum goudotii) entre 3050 y $3250 \mathrm{~m}$.

Entre 2500 y $3110 \mathrm{~m}$ se establecen los bosques de Quercus humboldtii, alianza fitosociológica (Monotropo unifloraeQuercion humboldtii) que incluye a los bosques de Quercus humboldtii con Clusia minor (Clusio minoris-Quercetum humboldtii) entre 2500-2800 m y a los de Quercus humboldtii con Weinmannia magnifolia (Weinmannio magnifoliaeQuercetum humboldtii) entre 2940 y $3110 \mathrm{~m}$.

En la vertiente Occidental en la región de vida subandina entre 1125 y 2400 (2600) $\mathrm{m}$ se establecen los bosques de Palicourea angustifolia y Hedyosmum racemosum (Palicoureo angustifoliae-Hedyosmion racemosi) que incluye a los bosques de Ocotea discolor y Huertea glandulosa (Ocoteo discoloris-Huerteetum glandulosae) entre $1000 \mathrm{y}$ $1800 \mathrm{~m}$ y a los de Brunellia occidentalis y Morus insignis (Brunellio occidentalis- Moretum insignis) entre 1850 y 2400-2600 m.

En la vertiente Oriental, entre 1400 y 2400 m se establecen los bosques de Hedyosmum racemosum y Nectandra caucana alianza fitosociológica (Hedyosmo racemosi-Nectandrion caucanae) entre 1400 y $2500 \mathrm{~m}$, donde se agruparon a los bosques de Chrysochlamys dependens y Nectandra globosa (Chrysochlamydo dependentis-Nectandretum globosae) entre 1400 y $1760 \mathrm{~m}$ y a los bosques de lauraceas con Nectandra acutifolia y Nectandra caucana (Nectandretum acutifoliocaucanae) entre 1750 y $2500 \mathrm{~m}$.

En la región de vida tropical entre 300 (vertiente Oriental) y $1000 \mathrm{~m}$ (vertiente Occidental), se establecen los bosques de Casearia corymbosa y Randia aculeata, formación fitosociológica del Caseario corymbosae-Randion aculeatae, con los bosques de Amyris pinnata y Croton glabellus (Amyro pinnatae-Crotonetum glabellae) a $1000 \mathrm{~m}$ en la vertiente Occidental y los de Mayna suaveolens y Casearia corymbosa (Mayno suaveolentis-Casearietum corymbosae) entre 300 y $600 \mathrm{~m}$ en la Oriental. También se presenta en esta región de vida y parcialmente en franjas de la región de vida subandina en la vertiente Oriental, la formación de los bosques de Acalypha villosa y Carludovica palmata de la alianza fitosociológia (Carludovico palmatae-Acalyphion villosae) entre 600 y $1400 \mathrm{~m}$ con los bosques de Ardisia foetida y Cupania latifolia (Ardisio foetidae-Cupanietum latifoliae) entre 600 y $1050 \mathrm{~m}$ y los de Protium macrophyllum y Rheedia madruno (Protio macrophyllae-Rheedietum madruñae) entre 1050 y $1400 \mathrm{~m}$.

\section{Cordillera Oriental (Macizo del Sumapaz)}

Los bosques y selvas entre 470 y $3500 \mathrm{~m}$ en las dos vertientes del macizo, se agrupan en la gran formación (clase fitosociológica) Palicoureo leuconerae- Cybianthetea iteoidis que incluye dos formaciones a nivel de orden fitosociológico:

Orden uno (1) Alchorneo glandulosae-Cybianthetalia iteoidis entre 470 y $2100 \mathrm{~m}$ en las dos vertientes, que engloba en la vertiente Occidental a la alianza fitosociológica Astronio graveolentis-Malpighion glabrae entre 470 y 1625 m, con los bosques de Randia acuminata y Machaerium capote (Randio acuminatae-Machaerietum capotae) entre 470 y 650; los bosques de Cordia polycephala - Matayba cf. elegans (Cordio polycephalae-Mataybetum elegantis) entre 900 y $1050 \mathrm{~m}$ y los bosques de Oreopanax acerifolius (Anthurio crassinerviOreopanecetum acerifolii) entre 1540 y $1625 \mathrm{~m}$.

También se encuentra en esta vertiente (Occidental), la formación de los boques de roble de Alchornea glandulosa y Quercus humboldtii (Alchorneo glandulosae-Quercetum humboldtii) entre 1200 y $2100 \mathrm{~m}$ de altitud. Otros tipos de bosque que igualmente se establecen en esta vertiente, son los de Mauria biringo (Thibaudio rigidiflorae-Maurietum cf., birringae) entre 2180 y 2300 m; el palmar mixto de Ceroxylon alpinum y Calatola costaricensis a $2450 \mathrm{~m}$; los bosques de Clusia elliptica e Ilex pernervata (Clusio elipticae-Ilicetum pernervati) entre 2730 y $3300 \mathrm{~m}$ y los bosques de Polylepis quadrijuga y Weinmannia reticulata a $3500 \mathrm{~m}$.

En la vertiente Oriental se establece la alianza fitosociológica Chamaedoreo pinnatifrondis-Matision cornucopiae entre 930 y $2450 \mathrm{~m}$ donde se agrupan a los bosques de Corytoplectus capitatus y Matisia cornu-copiae (Coryctoplecto capitatiMatisietum cornucopiae) entre 930 y $1900 \mathrm{~m}$ y a los bosques de Drimys granadensis y Weinmannia rollottii (Drimo granadensis-Weinmannietum rollotii) entre 2100 y $2450 \mathrm{~m}$. Entre 550 y $700 \mathrm{~m}$ se encuentran los bosques tropicales de Cassia moschata (Costo-Cassietum moschatae).

Orden dos (2) Clusio magnifoliae-Cybianthetalia iteoides en la vertiente Oriental, entre 2900 y 3800 m con la formación (alianza fitosociológica) Neurolepido apertae-Miconion jahnii entre 2900 y $3800 \mathrm{~m}$, donde se agrupan los bosques de Weinmannia reticulata y Weinmannia rollottii entre 2900 y $3100 \mathrm{~m}$ y los bosques de Neurolepis aperta y Myrsine dependens (Neurolepido apertae-Myrsinetum dependentis) entre 3200 y $3800 \mathrm{~m}$.

\section{Ecosistemas}

\section{Cordillera Occidental - Macizo del Tatamá (Van der Hammen, 2005).}

\section{Vertientes Occidental y Oriental}

Ecosistema de los palmares mixtos de Calatola costaricensis y Wettinia kalbreyeri (Calatolo-Wettinion) entre 1350 y $2500 \mathrm{~m}$ de altitud. 
Ecosistema de los palmares mixtos de Geonoma weberbaueri y Drimys granadensis (Geonomo-Drimion) entre $2500 \mathrm{y}$ $3200 \mathrm{~m}$ de altitud.

Ecosistema de los bosques achaparrados de Ilex suprema y Desfontainia parvifolia (complejo de ecosistemas del Desfontainio-Ilicion) entre 3300 y $3700 \mathrm{~m}$ de altitud.

Ecosistema de los bosques de Clusia clusioides y Clethra fagifolia (Clusio-Clethretum) entre 3300 y $3500 \mathrm{~m}$ de altitud.

Ecosistema de los bosques achaparrados de Miconia gleasoniana y Weinmannia engleriana (Weinmannio englerianae-Miconietum) entre 3520 y 3700 m de altitud.

\section{Vertiente Occidental.}

Ecosistema de los bosques de Cecropia sp. y Brosimum utile (Cecropio-Brosimion utilis) entre 450 y $1250 \mathrm{~m}$ de altitud.

Ecosistema de los bosques de Ocotea calophylla y Clusia multiflora (Ocoteo-Clusion) entre 2500 y $2860 \mathrm{~m}$ de altitud.

Ecosistema de los bosques enanos de Schefflera bejucosa y Miconia latifolia (Schefflero-Miconietum) entre 3300 y $3400 \mathrm{~m}$

\section{Vertiente Oriental.}

Ecosistema de los bosques de Ficus hartwegii con Callicarpa acuminata (Callicarpo-Ficetum) entre 1150 y $1700 \mathrm{~m}$ de altitud.

Ecosistema de los bosques de Quercus humboldtii con Pouteria lucuma (Pouterio-Quercetum) entre 2100 y $2500 \mathrm{~m}$.

\section{Cordillera Central-Parque Nacional Natural Los Nevados (Van der Hammen, 2003)}

\section{Vertientes Occidental y Oriental}

Ecosistema de los bosques de Casearia corymbosa y Randia aculeata (Caseario-Randion) entre 300 y 1100 m de altitud.

\section{Vertiente Occidental.}

Ecosistema de los bosques de Palicourea angustifolia y Hedyosmum racemosum (Palicoureo-Hedyosmion) entre 1125 y $2800 \mathrm{~m}$.

Ecosistema de los bosques de Weinmannia rollottii con Chusquea scandens (Chusqueo-Weinmannion rollottii) entre 2800 y $3050 \mathrm{~m}$.

Ecosistema de los bosques de Oreopanax nitidus y Neurolepis aristata (Neurolepido-Oreopanacion) entre 3400 y $3700 \mathrm{~m}$.

\section{Vertiente Oriental.}

Ecosistema de los bosques de Acalypha villosa y Carludovica palmata (Carludovico-Acalyphion) entre 600 y $1400 \mathrm{~m}$ de altitud.
Ecosistema de los bosques de Hedyosmum racemosum y Nectandra caucana (Hedyosmo-Nectandrion) entre 1400 y $2500 \mathrm{~m}$ de altitud.

Ecosistema de los boques de Quercus humboldtii y Monotropa uniflora (Monotropo-Quercion) entre $1800 \mathrm{y}$ $2600 \mathrm{~m}$ de altitud.

Ecosistema de los bosques de Clethra rugosa y especies de Weinmannia (Clethrion) entre 2800 y $3300 \mathrm{~m}$.

Ecosistema de los bosques de Hesperomeles lanuginosa y Diplostephium bicolor (Diplostephio-Hesperomelion) entre 3300 y $3750 \mathrm{~m}$

\section{Cordillera Oriental-Macizo del Sumapaz (Van der} Hammen, 2008).

\section{Vertiente Occidental}

Ecosistema de los bosques de Astronium graveolens y Malpighia glabra (Astronio-Malpighion) entre 400 y 1750 $\mathrm{m}$ de altitud.

Ecosistema de los bosques de Quercus humboldtii con Alchornea glandulosa (Quercion) entre 1200 y 2100 m.

Ecosistema de los bosques de Mauria biringo y Thibaudia rigidiflora (Thibaudio-Maurietum) entre 2050 y 2400 m.

Ecosistema de los palmares mixtos de Calatola costaricensis y Ceroxylon alpinum a $2475 \mathrm{~m}$.

Ecosistema de los bosques de Clusia elliptica e Ilex pernervata (Clusio-Iliceretum) entre 2730 y 3300 m.

Ecosistema de los bosques de Polylepis quadrijuga y Weinmannia reticulata a $3500 \mathrm{~m}$.

\section{Vertiente Oriental}

Ecosistema de los bosques de Cassia moschata y Costus sp. (Costo-Cassietum) entre 550 y $700 \mathrm{~m}$ de altitud.

Ecosistema de los bosques de Matisia cornu-copiae y Corytoplectus capitatus (Corytoplecto-Matisietum) entre 930 y $1900 \mathrm{~m}$.

Ecosistema del bosque-palmar de Matisia cornu-copiae y Chamaedorea pinnatifrons (Chamaedoreo-Matision) entre 930 y $2450 \mathrm{~m}$.

Ecosistema de los bosques de Drimys granadensis y Weinmannia rollottii (Drimo-Weinmannietum rollotii) entre 2100 y $2450 \mathrm{~m}$.

Ecosistema de los bosques de Weinmannia reticulata y Weinmannia rollottii entre 2900 y 3100 m.

Ecosistema de los bosques achaparrados de Miconia jahnii y Neurolepis aperta entre 2900 y 3500 m.

Distribución de la precipitación y de la riqueza de las plantas con flores en el gradiente altitudinal

Hay diferencia en los montos anuales de lluvia según la vertiente y las regiones de vida. Las vertientes exteriores 
en la cordillera Occidental (costado que mira al Pacífico) y Oriental (costado que mira a los llanos) y en la central (vertiente expuesta al río Magdalena), tienen valores mayores que los de las vertientes interiores. Entre las particularidades de la distribución de las lluvias, cabe resaltar los muy altos valores en las regiones de vida tropical y andina de la vertiente Occidental de la cordillera Occidental. En general hay tendencia a que la región de vida subandina $(>1100$ - 2200- $2400 \mathrm{~m})$ presente los valores mayores de lluvia, excepto en la vertiente Occidental de la cordillera Occidental (Tatamá). En la región de vida tropical, la temperatura máxima es $32.2^{\circ} \mathrm{C}$, la mínima 15.2 y la media 22.4. Obviamente con el progreso en altitud disminuyen los valores y en la región Andina se presenta temperatura máxima de 18.6, mínima de 4 y promedio de 11.5. Los tipos de clima según Thornthwaite incluyen las clases seco, semihúmedo $\mathrm{y}$ muy húmedo hasta los muy húmedos $\mathrm{y}$ superhúmedos de la media y alta montaña (Rangel et al., 2005c; Rangel y Arellano, 2008).

En los tres macizos considerados, el mayor número de especies de plantas con flores (1603) se encontró en el P.N.N. Los Nevados. La mayor expresión de la riqueza vegetal (600 especies) se encontró en la región subandina, seguida por la andina (490 especies) y por la tropical (120 especies). En el macizo del Tatamá (c. Occidental) se encontraron 1586 especies de plantas con flores, de las cuales la mayoría (697) se concentraban en la región subandina. En el Macizo de Sumapaz (c. Oriental) se registraron 1501 especies de plantas con flores, de las cuales la mayoría (921) crecían en la región subandina. Esta condición (concentración de la riqueza de plantas con flores) se asocia directamente con la mayor precipitación, no obstante que en esta región de vida(subandina), la pérdida de la cobertura vegetal (bosques originales) alcanza valores exageradamente altos (Rangel, 2000b, 2005/2006).

\section{Región del páramo}

Riqueza de las plantas con flores: La presentación de los resultados y la discusión se hacen en torno a la condición local (Colombia), que se compara con la de todos los países de la región biogeográfica, excepto Bolivia. Como fuente de referencia para las comparaciones figuran las contribuciones de Luteyn (1999), quien registró 3045 especies de plantas con flores para toda la región biogeográfica del páramo y de Rangel (2000c) quien mencionó para Colombia 3379 especies, cifra que se ha reducido a 3173 especies. Al combinar los dos inventarios se alcanza un número aproximado de 4696 especies. Sklenar et al. (2005) estimaron en 3595 el número de especies de plantas con flores para toda la región. Tomando en cuenta las diferencias de nuestras estimaciones en la riqueza de familias importantes con las de Sklenar et al. (2005), podríamos acercarnos a una cifra ponderada de 3700 especies para el páramo global desde Costa Rica hasta el Perú. En la Tabla 6 se enumeran las familias y los géneros con mayor riqueza en Colombia y en toda la región biogeográfica.

La revisión más reciente de las cifras mencionadas por Rangel (2000c) permitió documentar la presencia de 3173 especies de plantas con flores de 566 géneros y 118 familias, de las cuales las que presentan mayor número de géneros y especies son Asteraceae (100 géneros / 711 especies), Orchidaceae (57/580), Poaceae (40/148), Melastomataceae (12/112) y Bromeliaceae (7/98). En el páramo colombiano, Orchidaceae es la segunda familia más rica con 580 especies, por el contrario para toda el área del páramo tiene valores bajos en la lista de Sklenar et al. (2005). Otros valores altos en Colombia se presentan en Bromeliaceae, Apiaceae, Rubiaceae, Lamiaceae y Piperaceae.

Los géneros con mayor número de especies son Miconia, Diplostephium, Espeletia, Monticalia, Hypericum y Senecio. Géneros de Orquídeas como Epidendrum y Pleurothalis muestran en el páramo colombiano valores altos en comparación con los del páramo global (Sklenar et al. 2005).

El patrón de riqueza a nivel de familia que se detectó en las 20 familias mejor representadas significa el $78 \%$ de la riqueza en el páramo colombiano y el $69 \%$ en el páramo global, mientras que el patrón de los 28 géneros más ricos alcanza el 29 y $31 \%$ respectivamente.

Riqueza en otros grupos de la flora y de la fauna (varios autores en Rangel-Ch., ed., 2000a): En helechos se encontraron registros de 317 especies que significan el 21\% de la riqueza del grupo en Colombia. En hepáticas se poseen registros de 428 especies, $51 \%$ de la riqueza del país; en musgos de 457 especies, $47 \%$ de la riqueza del país y en líquenes de 361 especies, 23\% de la riqueza del país.

En la fauna, los grupos con mayor número de especies son los mamíferos con 70 especies y los anfibios con 90 especies que representan el 14 y el $13 \%$ de la riqueza del país. Se cuenta con registros de 154 especies de aves, $8 \%$ de la riqueza del país. El grupo más pobremente representado es el de los reptiles con 16 especies, $3 \%$ de la riqueza del país.

Tipos de vegetación: Se estima en 295 los tipos de vegetación (Rangel, 2000c; Pinto, 2010; Pinto y Rangel, 2010a), con composición florística y distribución geográfica definida. Predominan los matorrales (96 tipos), los pajonales (42), los prados (40) y los frailejonales (32). Otros tipos incluyen a los bosques achaparrados (16), los chuscales (15), rosetales (6) y a la vegetación de las turberas, pantanos y acuática (48). El esbozo de la base de datos sobre la vegetación del páramo de Colombia y un ejemplo del enfoque fitosociológico con el esquema sintaxonómico que permitirá conocer en definitiva el arreglo de las comunidades vegetales del páramo, según la composición florística fue recientemente publicado por Rangel y Pinto (2012). 
Tabla 6. Familias y géneros de plantas con flores más ricos en el área biogeográfica del páramo y en la región paramuna de Colombia.

\begin{tabular}{|c|c|c|}
\hline & \\
\hline & $\begin{array}{c}\text { Colombia (Rangel } \\
\text { 2000c, 2006) }\end{array}$ & $\begin{array}{c}\text { Páramo global (Sklenar } \\
\text { et al., 2005) }\end{array}$ \\
\hline Familia & Géneros/especies & Géneros/especies \\
\hline Asteraceae & $100 / 711$ & $113 / 973$ \\
\hline Orchidaceae & $57 / 580$ & $27 />100$ \\
\hline Poaceae & $40 / 148$ & $47 / 231$ \\
\hline Melastomataceae & $12 / 112$ & $9 / 113$ \\
\hline Bromeliaceae & $7 / 98$ & $6 / 88$ \\
\hline Ericaceae & $18 / 88$ & $15 / 79$ \\
\hline Scrophulariaceae & $19 / 86$ & $14 / 140$ \\
\hline Apiaceae & $19 / 73$ & $15 / 62$ \\
\hline Cyperaceae & $20 / 69$ & $8 / 74$ \\
\hline Rubiaceae & $15 / 68$ & $6 / 25$ \\
\hline Fabaceae & $13 / 65$ & $9 / 77$ \\
\hline Rosaceae & $11 / 62$ & $10 / 76$ \\
\hline Lamiaceae & $10 / 53$ & $8 / 42$ \\
\hline Gentianaceae & $5 / 50$ & $4 / 94$ \\
\hline Solanaceae & $10 / 47$ & $8 / 59$ \\
\hline Hypericaceae & $1 / 46$ & $1 / 56$ \\
\hline Brassicaceae & $11 / 46$ & $14 / 73$ \\
\hline Piperaceae & $2 / 37$ & $2 / 20$ \\
\hline Campanulaceae & $6 / 30$ & $6 / 23$ \\
\hline Geraniaceae & $2 / 26$ & $3 / 49$ \\
\hline $\begin{array}{l}\text { Suma de las (20) } \\
\text { familias más ricas }\end{array}$ & $2495(78 \%)$ & $2454(69 \%)$ \\
\hline Resto de familias & $678(22 \%)$ & $1141(31 \%)$ \\
\hline
\end{tabular}

\begin{tabular}{|c|c|c|}
\hline \multirow[t]{2}{*}{ Género } & Colombia & $\begin{array}{l}\text { Sklenar et al., } \\
\quad \text { (2005) }\end{array}$ \\
\hline & \multicolumn{2}{|c|}{ Especies } \\
\hline Epidendrum & 65 & $(>27) 65$ \\
\hline Miconia & 61 & 54 \\
\hline Diplostephium & 59 & 73 \\
\hline Espeletia & $60^{*}$ & $67-77$ \\
\hline Pleurothallis & 50 & $(>15) 50$ \\
\hline Monticalia & 47 & 111 \\
\hline Hypericum & 46 & 56 \\
\hline Senecio & 43 & 67 \\
\hline Lupinus & 38 & 56 \\
\hline Solanum & 30 & 43 \\
\hline Ageratina & 28 & 40 \\
\hline Baccharis & 28 & 52 \\
\hline Carex & 27 & 32 \\
\hline Weinmannia & 27 & 23 \\
\hline Gynoxys & 25 & 49 \\
\hline Valeriana & 25 & 58 \\
\hline Geranium & 24 & 46 \\
\hline Lachemilla & 24 & 34 \\
\hline Calamagrostis & 21 & 35 \\
\hline Calceolaria & 16 & 65 \\
\hline $\begin{array}{l}\text { Suma de los }(20) \\
\text { géneros más ricos }\end{array}$ & $744(23 \%)$ & $1086(30 \%)$ \\
\hline Total general & 3173 & 3595 \\
\hline
\end{tabular}

*Diazgranados, 2012.

\section{Ecosistemas}

Cordillera Occidental (Macizo del Tatamá, con base en Pinto y Rangel, 2010b)

\section{Vertientes Occidental y Oriental}

Ecosistema del pajonal de Calamagrostis effusa y Rhynchospora aristata entre 3500 y $3550 \mathrm{~m}$ de altitud.

Ecosistema del chuscal-matorral de Chusquea tessellata con Diplostephium schultzii entre 3420 y 3670 m.

Ecosistema del chuscal-matorral de Chusquea tessellata con Diplostephium rupestre entre 3400 y 4100 m.

Ecosistema del pajonal de Calamagrostis effusa y Sphagnum magellanicum entre 3600 y 3770 m.

Cordillera Central (Parque Nacional Natural Los Nevados, Van der Hammen, 2003)

\section{Vertientes Occidental y Oriental}

Ecosistema del pastizal-frailejonal de Calamagrostis effusa, Calamagrostis recta y Espeletia hartwegiana (Espeletio-
Calamogrostietalia effuso rectae) entre 3900 y 4200 m de altitud.

Ecosistema de los pajonales de Festuca dolichophylla y Calamagrostis effusa (Festuco-Calamagrostion effusae) entre 4000 y $4200 \mathrm{~m}$.

Ecosistema del pastizal-herbazal de Cerastium subspicatum y Calamagrostis recta (Cerastio-Calamagrostion rectae) entre 3900 y $4250 \mathrm{~m}$.

Ecosistema del pajonal de Festuca breviaristata y Agrostis araucana (Festuco-Agrostion) entre 4300 y 4470 m.

Ecosistema del pastizal-herbazal de Agrostis foliata y Cerastium floccosum (Agrostio-Cerastion) entre 4150 y $4600 \mathrm{~m}$.

Cordillera Oriental (Macizo de Sumapaz, Van der Hammen, 2008)

\section{Vertientes Occidental y Oriental}

Ecosistema del matorral de Baccharis macrantha y Rumex tolimensis (Rumico-Baccharidetum) entre 3600 y $3650 \mathrm{~m}$ de altitud. 
Ecosistema de las plantas en cojines y rosetas basales de Oreomyrrhis andicola y Azorella multifida (OreomyrridoAzorellion) entre 400 y $4200 \mathrm{~m}$ de altitud.

Ecosistema de los frailejonales de Espeletia grandiflora con Rhynchospora macrochaeta (Rhynchosporo-Espeletion grandiflorae) entre 3300 y $4200 \mathrm{~m}$ de altitud.

Ecosistema del chuscal-matorral de Chusquea tessellata con Hypericum lancioides (ecosistema del complejo de Hypericum y Chusquea) entre 3150 y $4000 \mathrm{~m}$ de altitud.

La distribución de la riqueza de las familias de las plantas con flores en los países de la región biogeográfica del páramo

En la Tabla 7 se muestran los valores del número de especies en las familias más ricas en la flora paramuna en los países que abarca su distribución geográfica. El país con mayor número de especies es Colombia (3173), seguido por Ecuador (1678). El país con menor representación es Panamá con 200 especies. Las familias más ricas en especies son Asteraceae, Orchidaceae, Poaceae y Melastomataceae. El patrón de riqueza de las diez familias más ricas supera en todos los casos más del $50 \%$, con lo cual se configura un carácter bastante distintivo para esta flora de alta-montaña.

A nivel de géneros (Tabla 8) Monticalia (Pentacalia), Lupinus, Hypericum, Miconia, Carex, Agrostis y Diplostephium son los más ricos en todos los países, con una mayor expresión en el núcleo de páramos de Colombia, Venezuela y Ecuador. Otro grupo de géneros conformados por Calceolaria,
Valeriana, Gentianela, Poa, Calamagrostis y Ageratina, presentes en todos los países muestran valores mayores en el núcleo de páramos del Sur (Ecuador y Perú). Espeletia alcanza su máxima riqueza en los páramos colombianos y está presente también en Venezuela y en Ecuador.

\section{Riqueza-distintividad de las plantas con flores del páramo según países}

La mayor concentración de especies 3173 (86\% de la riqueza total del páramo) se presenta en Colombia (16204 $\mathrm{Km}^{2}$ de superficie); le sigue Ecuador $\left(20000 \mathrm{Km}^{2}\right)$ con 1678 especies, que representan el $45 \%$ de la riqueza global. En distintividad biológica, la condición nacional está relacionada con la relación entre el número de especies restringidas y la riqueza a nivel de país. La mayor expresión de la distintividad biológica se presenta en los páramos de Colombia, $47 \%$ a nivel nacional y $41 \%$ a nivel global, seguido por Ecuador $42 \%$ a nivel nacional y $45 \%$ a nivel global (Rangel, 2006).

Cuando se comparan los inventarios de las plantas con flores de los diferentes países, los valores más altos en cuanto a similitud se encuentran entre Colombia y Venezuela, que comparten 840 especies (16\%); Colombia y Ecuador comparten 777 especies (15\%). En la condición EcuadorPerú-Colombia, páramos del Sur, se comparten 333 especies (6\%), valor mayor que la condición Colombia-Costa RicaPanamá páramo del Norte, con 48 especies $(0.9 \%)$. Entre Colombia y Perú se comparten 430 especies $(8.3 \%)$ y entre Ecuador-Perú 493 especies (9\%).

Tabla 7. Patrón de la riqueza según familias en los diferentes países de la región biogeográfica del páramo

\begin{tabular}{|c|c|c|c|c|c|c|c|}
\hline & Costa Rica & Panamá & Colombia & Venezuela & Ecuador & Perú & Páramo global \\
\hline & 72 familias & 54 familias & 118 familias & 95 familias & 92 familias & 103 familias & 127 familias \\
\hline Familias & Especies & Especies & Especies & Especies & Especies & Especies & Especies \\
\hline Asteraceae & 73 & 25 & 711 & 261 & 339 & 282 & 973 \\
\hline Orchidaceae & 18 & 3 & 580 & 200 & 123 & 23 & 101 \\
\hline Poaceae & 52 & 21 & 148 & 179 & 148 & 121 & 231 \\
\hline Melastomataceae & 8 & 2 & 112 & 49 & 82 & 24 & 113 \\
\hline Bromeliaceae & 7 & 5 & 98 & 42 & 23 & 28 & 88 \\
\hline Ericaceae & 12 & 7 & 88 & 32 & 43 & 14 & 79 \\
\hline Scrophulariaceae & 18 & 12 & 86 & 34 & 75 & 91 & 140 \\
\hline Cyperaceae & 19 & 10 & 69 & 39 & 44 & 29 & 74 \\
\hline Rubiaceae & 11 & 8 & 68 & 26 & 31 & 14 & 25 \\
\hline Apiaceae & 16 & 10 & 73 & 29 & 34 & 23 & 62 \\
\hline Suma de las 10 familias & $234(56 \%)$ & $103(51 \%)$ & $2033(64 \%)$ & $891(62 \%)$ & $942(56 \%)$ & $679(53 \%)$ & $1886(52 \%)$ \\
\hline Resto & 182 & 97 & 1140 & 546 & 736 & 558 & 1814 \\
\hline Totales & 416 & 200 & 3173 & 1437 & 1678 & 1207 & 3700 \\
\hline
\end{tabular}

Fuentes: Brako y Zaruchi (1993); Jorgensen y León (1999); Luteyn (1999), Dorr et al. (2000), Vargas y Sánchez (2005), Briceño y Morillo (2002, 2006); Rangel (2000c, 2006). 
Tabla 8. Riqueza a nivel genérico en los países del área biogeográfica del páramo.

\begin{tabular}{|c|c|c|c|c|c|c|c|}
\hline & $\begin{array}{c}\text { Costa Rica } \\
216 \mathrm{~g} \\
416 \mathrm{sp}\end{array}$ & $\begin{array}{c}\text { Panamá } \\
133 \text { g } \\
200 \mathrm{sp}\end{array}$ & $\begin{array}{c}\text { Colombia } \\
572 \text { g } \\
3173 \text { sp }\end{array}$ & $\begin{array}{l}\text { Venezuela } \\
390 \mathrm{~g} \\
1437 \mathrm{sp}\end{array}$ & $\begin{array}{c}\text { Ecuador } \\
388 \mathrm{~g} \\
1678 \mathrm{sp}\end{array}$ & $\begin{array}{c}\text { Perú } \\
376 \text { g } \\
1207 \text { sp }\end{array}$ & $\begin{array}{c}\text { Páramo } \\
\text { global }\end{array}$ \\
\hline Monticalia (Pentacalia) & 2 & 2 & 47 & 35 & 16 & 6 & 111 \\
\hline Lupinus & 3 & 2 & 38 & 16 & 26 & 10 & 56 \\
\hline Hypericum & 8 & 3 & 46 & 21 & 16 & 10 & 56 \\
\hline Miconia & 3 & 1 & 61 & 22 & 51 & 12 & 54 \\
\hline Carex & 8 & 2 & 27 & 16 & 17 & 12 & 32 \\
\hline Agrostis & 5 & 1 & 18 & 19 & 14 & 8 & 24 \\
\hline Gaultheria & 2 & 2 & 14 & 10 & 15 & 6 & 19 \\
\hline Gnaphalium & 6 & 2 & 16 & 11 & 11 & 6 & 18 \\
\hline Diplostephium & 1 & 1 & 59 & 10 & 22 & 13 & 73 \\
\hline Lachemilla & 6 & 2 & 24 & 21 & 24 & 16 & 34 \\
\hline Espeletia & 0 & 0 & 60 & 13 & 1 & 0 & 67 \\
\hline Senecio & 5 & 1 & 43 & 5 & 10 & 98 & 67 \\
\hline Calceolaria & 6 & 2 & 16 & 7 & 35 & 50 & 65 \\
\hline Valeriana & 3 & 2 & 25 & 15 & 25 & 38 & 58 \\
\hline Gentianela & 0 & 0 & 10 & 2 & 28 & 55 & 51 \\
\hline Poa & 4 & 2 & 9 & 8 & 10 & 33 & 20 \\
\hline Calamagrostis & 3 & 2 & 21 & 22 & 21 & 40 & 35 \\
\hline Baccharis & 0 & 0 & 28 & 8 & 27 & 20 & 52 \\
\hline Ageratina & 8 & 6 & 28 & 9 & 17 & 12 & 40 \\
\hline Festuca & 8 & 4 & 14 & 11 & 12 & 35 & 40 \\
\hline Oritrophium & 0 & 0 & 3 & 8 & 5 & 5 & 19 \\
\hline Ranunculus & 4 & 1 & 13 & 8 & 10 & 7 & 16 \\
\hline Rubus & 5 & 2 & 16 & 3 & 13 & 8 & 14 \\
\hline Castilleja & 4 & 3 & 9 & 6 & 7 & 8 & 13 \\
\hline Chusquea & 5 & 2 & 11 & 3 & 4 & 2 & 12 \\
\hline Rhynchospora & 5 & 3 & 12 & 9 & 2 & 2 & 10 \\
\hline Vaccinium & 4 & 3 & 3 & 3 & 2 & 2 & 8 \\
\hline Suma de los géneros mas ricos & $108(26 \%)$ & $51(29 \%)$ & $671(21 \%)$ & $321(22 \%)$ & $641(38 \%)$ & $514(42 \%)$ & \\
\hline
\end{tabular}

Fuentes: Luteyn (1999), Dorr et al. (2000), Jorgensen y León (1999), Brako y Zaruchi (1993), Briceño y Morillo (2002, 2006), Rangel (2000c), Sklenar et al. (2005), Diazgranados (2012).

Tipos de páramos según los montos de la precipitación en Colombia.

Rangel (2000b) con base en la caracterización de los montos anuales de precipitación en 80 estaciones climatológicas, propuso las siguientes clases de páramos en Colombia: Pluviales: monto anual de precipitación $>4061 \mathrm{~mm}$, localidad representativa Torre de San Francisco, macizo colombiano departamento del Putumayo. Superhúmedo: monto anual de precipitación entre $2919 \mathrm{~mm}$ y $3492 \mathrm{~mm}$, localidad representativa, La Cumbre en el departamento de Antioquia, cordillera Central. Muy húmedos: monto anual de precipitación entre $2345 \mathrm{~mm}$ y $2918 \mathrm{~mm}$, localidad representativa Chuza en la cordillera Oriental departamento de Cundinamarca. Húmedos: monto anual de precipitación entre $1771 \mathrm{~mm}$ y $2344 \mathrm{~mm}$, localidad representativa Laguna San Rafael departamento del Cauca. Semihúmedos: monto anual de precipitación entre $1197 \mathrm{~mm}$ y $1770 \mathrm{~mm}$, localidad representativa Palacios Guasca en la cordillera Oriental departamento de Cundinamarca. Secos: monto anual de precipitación entre $623.5 \mathrm{~mm}$ y $1196.5 \mathrm{~mm}$, localidad representativa Boqueron departamento de Tolima, cordillera Central y Berlín departamento de Santander, cordillera Oriental con $623.5 \mathrm{~mm}$, probablemente uno de los valores más bajos en todo el páramo colombiano. 


\section{La precipitación y la distribución de la riqueza en las franjas del páramo colombiano}

Los valores mayores en la riqueza en plantas con flores (1783), en los tipos de vegetación (75) y en los grupos de la fauna como aves (117), en anfibios (49) y en mamíferos (44), junto con mayor valor en el monto anual de precipitación (1716 mm), se presentan en el subpáramo (3201-3500 m de altitud). Los menores valores (455 especies) de plantas con flores, 87 tipos de vegetación, aves (46), anfibios (5) y mamíferos (1), junto con un monto anual de precipitación de $1229 \mathrm{~mm}$, se presentan en el superpáramo, áreas con situaciones muy contrastes, extensas superficies desprovistas de vegetación y fluctuaciones marcadas de temperatura, congelación durante la noche y valores muy altos durante el día (Sturm y Rangel, 1985; Rangel, 2000b).

\section{Discusión - consideraciones finales}

Riqueza de la flora: Entre las regiones naturales de Colombia, la Andina o cordillerana es la que presenta mayor concentración de la biodiversidad con cerca de 11.500 especies de plantas con flores, 914 de musgos, 756 de hepáticas,
1396 de líquenes y 1050 de helechos. Le sigue la región de la Amazonía con cerca de 7.600 especies de plantas con flores, 510 de helechos, 322 de líquenes, 174 de musgos y cerca de 100 de hepáticas. En el Chocó biogeográfico se cuenta con 4525 especies de plantas con flores, además de 132 de musgos, 189 de líquenes, 170 de hepáticas y 425 de helechos. Los valores menores de riqueza, se presentan en la Orinoquia con 4347 especies de plantas con flores, 254 de helechos, 130 de líquenes y 86 de musgos.

En las plantas con flores (Tabla 9) las familias Orchidaceae (205 géneros / 3588 especies), Asteraceae (202/1420), Rubiaceae (109/1214), Melastomataceae (68/948) y Poaceae (183/907) muestran que el patrón de riqueza a nivel mundial, igualmente se repite en Colombia. Las 40 familias más ricas representan el $62.9 \%$ de la riqueza a nivel del país y a nivel del mundo $62.1 \%$.

Riqueza de la fauna: Los registros de 974 especies de aves, 484 de anfibios, 274 de reptiles y 329 de mamíferos posicionan a la región andina en el primer lugar. Le sigue en importancia, la región del Caribe con 917 especies de aves, 104 de anfibios, 198 de reptiles y 188 de mamíferos.

Tabla 9. Las familias de plantas con flores más ricas en Colombia y en el mundo

\begin{tabular}{llccc}
\hline & & \multicolumn{2}{c}{ Colombia } & Mundo \\
\cline { 2 - 5 } & Familias & Géneros & Especies & Especies \\
\hline 1 & Orchidaceae & 205 & 3588 & 30000 \\
\hline 2 & Asteraceae & 202 & 1420 & 20000 \\
\hline 3 & Rubiaceae & 109 & 1214 & 6500 \\
\hline 4 & Melastomataceae & 68 & 948 & 4000 \\
\hline 5 & Poaceae & 183 & $907^{*}$ & 11000 \\
\hline 6 & Araceae & 22 & $650 *$ & 1800 \\
\hline 7 & Piperaceae & 7 & 604 & 1700 \\
\hline 8 & Gesneriaceae & 34 & 520 & 2500 \\
\hline 9 & Fabaceae & 97 & 510 & 12000 \\
\hline 10 & Bromeliaceae & 22 & 502 & 2000 \\
\hline 11 & Euphorbiaceae & 75 & 438 & 7000 \\
\hline 12 & Solanaceae & 42 & 405 & 3000 \\
\hline 13 & Cyperaceae & 37 & 363 & 5000 \\
\hline 14 & Lauraceae & 15 & 350 & 2600 \\
\hline 15 & Mimosaceae & 32 & 345 & 2500 \\
\hline 16 & Annonaceae & 27 & 280 & 2300 \\
\hline 17 & Apocynaceae & 55 & 260 & 1600 \\
\hline 18 & Clusiaceae & 20 & 245 & 1200 \\
\hline 19 & Arecaceae & 44 & 231 & 1500 \\
\hline 20 & Malpighiaceae & 23 & 204 & 1200 \\
\hline 21 & Lamiaceae & 203 & 3200 \\
\hline & & & & \\
\hline
\end{tabular}

\begin{tabular}{llccc}
\hline & & \multicolumn{2}{c}{ Colombia } & Mundo \\
\hline & Familias & Géneros & Especies & Especies \\
\hline 22 & Acanthaceae & 32 & 202 & 2500 \\
\hline 23 & Caesalpiniaceae & 39 & 200 & 2700 \\
\hline 24 & Ericaceae & 23 & 200 & 4000 \\
\hline 25 & Sapindaceae & 18 & 200 & 1700 \\
\hline 26 & Bignoniaceae & 50 & 194 & 800 \\
\hline 27 & Myrtaceae & 15 & $165 *$ & 3000 \\
\hline 28 & Passifloraceae & 1 & 154 & 650 \\
\hline 29 & Scrophulariaceae & 33 & 130 & 3000 \\
\hline 30 & Sapotaceae & 12 & 130 & 800 \\
\hline 31 & Verbenaceae & 19 & 120 & 2600 \\
\hline 32 & Chrysobalanaceae & 6 & 116 & 525 \\
\hline 33 & Araliaceae & 4 & 105 & 1400 \\
\hline 34 & Convolvulaceae & 18 & 105 & 1700 \\
\hline 35 & Moraceae & 22 & 101 & 1000 \\
\hline 36 & Heliconiaceae & 1 & 100 & 100 \\
\hline 37 & Cactaceae & 26 & 82 & 2000 \\
\hline 38 & Lecythidaceae & 6 & 77 & 300 \\
\hline 39 & Apiaceae & 23 & 56 & 3000 \\
\hline 40 & Amaranthaceae & 14 & 49 & 900 \\
\hline & Suma 40 familias & & $16673(62,9 \%)$ & $155275(62,1 \%)$ \\
\hline & Total & & 26500 & 250000 \\
\hline & & & & \\
\hline
\end{tabular}

Fuentes: *Natalia Castaño; Carlos Parra (cp), Giraldo-C. (2011). 
Variabilidad climática: De las tierras bajas, cálidas o regiones extra-andinas, la que presenta valores mayores en precipitación es el Chocó biogeográfico, mientras que los valores menores se presentan en el Caribe. Los promedios mensuales de lluvia varían entre $437.2 \mathrm{~mm}$ en el Chocó, $259.9 \mathrm{~mm}$ en la Amazonia, hasta $185 \mathrm{~mm}$ en el Caribe, donde se presentan los valores mayores en temperatura media $\left(27.4^{\circ} \mathrm{C}\right)$, mientras que en las otras regiones los valores fluctúan entre $25.3^{\circ} \mathrm{C}$ en la Amazonia y $25.9^{\circ} \mathrm{C}$ en el Chocó biogeográfico. La evaporación $(\mathrm{mm})$ mensual es mayor en el Caribe $(166 \mathrm{~mm})$ y en el Chocó se presenta el valor más bajo con $24 \mathrm{~mm}$. Los tipos de clima según Thornthwaite que predominan en el Chocó y la Amazonia son húmedos, muy húmedos y superhúmedos. En la Orinoquia superhúmedos, húmedos y moderadamente húmedos y en el Caribe el mosaico es más variado con climas desde superhúmedos hasta áridos.

En el gradiente topográfico, la región subandina es la más húmeda y una característica distintiva, es que en las partes altas en la región del páramo en Colombia, los montos de precipitación son relativamente altos cuando se les compara con otras montañas neotropicales. En todas las regiones naturales de Colombia es muy clara la existencia de patrones latitudinales y altitudinales muy bien definidos en cada una de las regiones naturales.

\section{Riqueza de la vegetación}

En la Figura 1, se muestra de manera esquemática la riqueza a nivel beta o de los tipos de vegetación. De los cerca de 1200 tipos de vegetación descritos o mencionados en la literatura, en la región cordillerana (Andina) se concentra la mayor riqueza con 571 tipos. En el páramo hay una mayor variabilidad (295 tipos), resultado que probablemente se asocia con las variaciones en la fisiografía y en las

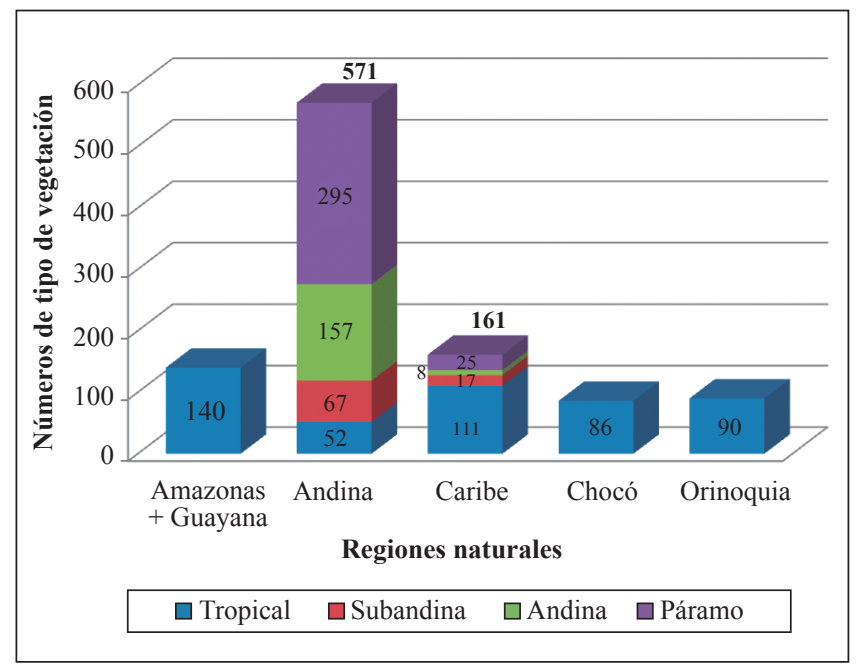

Figura 1. Distribución de los tipos de vegetación en las regiones naturales de Colombia condiciones climáticas. De las regiones naturales extraandinas, la más rica es el Caribe, en donde la presencia de macizos montañosos altos incrementa la riqueza de la vegetación de la planicie donde se presentan climas desde el árido (La Guajira) hasta los superhúmedos pluviales del Sur del departamento de Córdoba.

\section{Ecosistemas}

Para todo el país excepto la región amazónica, hasta ahora se han caracterizado 140 ecosistemas; en el Caribe (54) se presenta la mayor riqueza seguida por la región Andina o cordillerana (44), en la Orinoquia se definieron 22 y en el Chocó biogeográfico 20 .

\section{Significado de la Biodiversidad de Colombia}

\section{A nivel regional}

Los valores que se han registrado para las diferentes regiones naturales de Colombia son más altos que los de áreas geográficas similares, por ejemplo cuando comparamos la riqueza vegetal y animal de la Orinoquia con la de los llanos venezolanos o cuando solamente comparamos aspectos de la flora y la vegetación con áreas con características generales similares como las sabanas del Beni y del Espíritu santo en Bolivia o con las zonas de tierras bajas en áreas de Centroamérica como Costa Rica e inclusive México (Rangel \& Minorta, 2014).

El Chocó biogeográfico de Colombia es más rico que las áreas de esta región biogeográfica en Centroamérica y en el Ecuador.

Las áreas del Caribe de Colombia incluyen buena parte de la riqueza biológica de otros países de la cuenca como Venezuela e inclusive sus valores superan a los de diversos países del globo (Rangel, 2012b).

En la región paramuna de Colombia está representada el $85 \%$ de la riqueza de las plantas con flores, de la cual cerca del $41 \%$ está restringida en su distribución a Colombia.

\section{A nivel global.}

A nivel del globo, Colombia es uno de los dos países con mayor expresión de la biodiversidad. En las plantas con flores hay registros de aproximadamente 26.500 especies, que le sitúan a continuación de Brasil con 28.000. Los valores de riqueza en helechos (1600), musgos (976) y en líquenes (1.700), colocan a nuestro país como el más rico en cada uno de estos grupos en el concierto neotropical. La riqueza de los bosques y otros tipos de vegetación como selvas, matorrales, pastizales, rosetales, en Colombia alcanza cerca de 1.200 tipos diferentes, lo cual le confiere una característica singular como uno de los países con mayor variabilidad en esta condición a nivel mundial.

En la Tabla 10, se muestran los valores de biodiversidad en diferentes países calificados como megadiversos y a manera de comparación los valores de Colombia. Aunque 
Tabla 10. Riqueza en plantas con flores y en grupos de la fauna en los países megadiversos del globo.

\begin{tabular}{lcccccc}
\hline \multirow{2}{*}{ Pais } & \multirow{2}{*}{$\begin{array}{c}\text { Superficie } \\
\left(\mathbf{K m}^{2}\right)\end{array}$} & Plantas con flores & & \multicolumn{2}{c}{ Fauna } \\
\cline { 4 - 7 } & $1^{\prime} 141.748$ & Mamíferos & Aves & Reptiles & Anfibios \\
\hline Colombia & $1^{\prime} 285.216$ & 26500 & 492 & 1834 & 512 & 700 \\
\hline Perú & $1^{\prime} 919.443$ & 17200 & 508 & 1770 & 298 & 241 \\
\hline Indonesia & $1^{\prime} 958.201$ & $20-25000$ & 515 & 1683 & 511 & 270 \\
\hline México & $8^{\prime} 511.996$ & 23400 & 535 & 1098 & 804 & 361 \\
\hline Brasil & 270.670 & 28000 & 652 & 1786 & 468 & 502 \\
\hline Ecuador & $9^{\prime} 571.300$ & 16000 & 404 & 1607 & 404 & 464 \\
\hline China & 916.445 & 22000 & 510 & 1373 & 282 & 190 \\
Venezuela & 15820 & 351 & 1373 & 651 \\
\hline
\end{tabular}

Fuente: Venezuela; plantas con flores: Hokche \& Berry (2008), mamíferos: Ochoa \& Aguilera (2003), anfibios: La Marca (2003a), reptiles: La Marca (2003b). Indonesia; http://www.ran.org/indonesia_s_rainforests_biodiversity_and_endangered_species

en varios casos no hay documentos de acceso fácil que permitan confrontar los estimativos, al comparar los valores absolutos, Colombia alcanza mayores valores en los grupos de tetrapoda incluidos en nuestras investigaciones, que los otros países. Es el país más rico en aves (1834 especies) y en anfibios (700 especies) y alcanza valores muy importantes en otros grupos como reptiles (512 especies) y mamíferos (492 especies).

\section{Conclusiones-razones de la expresión alta en la biodiversidad}

Colombia es uno de los dos países del globo con mayor biodiversidad. Es el segundo en especies de plantas con flores. En helechos, en musgos y en líquenes es el país más rico a nivel neotropical.

Cerca 1.200 tipos diferentes de selvas, matorrales, pastizales, rosetales, le confieren característica singular como uno de los países con mayor variedad en hábitats a nivel mundial.

Es el país más rico en aves y en anfibios y alcanza valores muy importantes en reptiles y mamíferos.

En nuestras regiones naturales, la cordillerana o Andina es la que presenta mayor concentración de la biodiversidad.

La biodiversidad del Chocó biogeográfico y del páramo, no tienen comparación con la de otras regiones geográficas del mundo. El Chocó biogeográfico representa la mayor concentración de riqueza en flora de todos los biomas terrestres húmedos del globo.

El páramo colombiano representa el $60 \%$ de la riqueza de la flora del bioma de alta montaña de Centroamérica y el Norte de Suramérica.

Entre las razones que sirven para explicar esta expresión tan alta de riqueza, figuran: La posición geográfica (radiación) y la influencia de dos mares (precipitación).

La historia geológica (Paleogeno-Pleistoceno) que permitió la dispersión, intercambio y especiación de la biota
La variabilidad y la riqueza que significa cinco extensas regiones naturales continentales con heterogeneidad topográfica y variabilidad del hábitat muy altas.

La variabilidad climática en las regiones naturales: paisajes contrastantes, planicies y extensos valles separados por mesas, mesetas, sierras, serranías e imponentes macizos montañosos que se relacionan directamente con mosaico de climas, la mayoría calificados como húmedos.

A manera de ejemplo cabe retomar el caso de la región Caribe con sus extensas planicies aledañas a las orillas del mar y separadas por macizos y serranías que han significado la profusión de 14 unidades climáticas, desde las casi desérticas en la alta Guajira con menos de 250 $\mathrm{mm}$ de lluvia anuales, hasta las muy húmedas pluviales del Sur del departamento de Córdoba con montos superiores a los $3.000 \mathrm{~mm}$ anuales de lluvia. Este patrón topográfico, extensos valles y planicies separados por macizos montañosos se repite en todas nuestras regiones naturales, produciendo un mosaico de climas, la mayoría calificados como húmedos.

\section{Agradecimientos}

Al instituto de Ciencias Naturales, Facultad de Ciencias de la Universidad Nacional, donde ha transcurrido toda mi carrera docente. A mis profesores Thomas van der Hammen (+) y Helmut Sturm $(+)$ y a mis colegas A.M. Cleef, Jaime Aguirre y Olga Castaño, por su fabulosa colaboración de toda la vida. A mis colegas del grupo de investigación biodiversidad y conservación del Instituto de Ciencias Naturales por su constante compañía y apoyo que han sido decisivas para alcanzar logros importantes en la meta de liderar el inventario de la biodiversidad de Colombia. A mis estudiantes por sus inquietudes y por la estrecha colaboración que me ha permitido ayudarles en su formación y enriquecerme con sus opiniones y cuestionamientos. 


\section{Conflicto de intereses}

El autor declara no tener ningún conflicto de intereses.

\section{Bibliografía}

Acosta-G., A.R. 2000. Ranas, Salamandras y Caecilias (Tetrapoda: Amphibia) de Colombia. Biota Colombiana 1 (3): 289-319.

Avella-M., A. \& Rangel-Ch., J.O. 2012. Composición florística y aspectos estructurales de la vegetación boscosa del sur del departamento de Córdoba. En: J.O. Rangel-Ch. (ed.). Colombia Diversidad Biótica XII. La región Caribe de Colombia: 477-537. Universidad Nacional de Colombia Instituto de Ciencias Naturales, Bogotá D.C.

Avella-M., A. \& Rangel-Ch., J.O. 2014. Oak forests types of Quercus humboldtii in the Guantiva - La Rusia - Iguaque corridor (Santander-Boyacá, Colombia): Their conservation and sustainable use. Colombia Forestal 17 (1): 100-116.

Brako, L. \& Zarucchi, J.L. 1993. Catálogo de las angiospermas y gimnospermas del Perú. Monographs in Systematic Botany. Mo. Bot. Garden. 1286 pp. St. Louis, Missouri. USA.

Briceño, B. \& Morillo, G. 2002. Catálogo abreviado de las plantas con flores de los páramos de Venezuela Parte I. Dicotiledóneas (Magnoliopsida). Acta Botánica Venezuelica 25 (1): 1-46.

Briceño, B. \& Morillo, G. 2006. Catálogo de las plantas con flores de los páramos de Venezuela. Parte II. Monocotiledóneas (Liliopsida). Acta Botánica Venezuelica 29 (1): 89-134.

Cantillo, E.E. \& Rangel-Ch., J.O. 2011. Los patrones de la estructura y de la riqueza de la vegetación boscosa de Colombia-síntesis-. En: J.O. Rangel-Ch. (ed.). Colombia Diversidad Biótica XI. Patrones de la estructura y de la riqueza de la vegetación en Colombia: 435-461. Universidad Nacional de Colombia-Instituto de Ciencias Naturales, Bogotá D.C.

Cleef, A.M., Rangel-Ch., J.O., Van der Hammen, T. \& Jaramillo-M., R. 1984. La vegetación de las selvas del Transecto Buritaca, Sierra Nevada de Santa Marta. En: T. van der Hammen y P. Ruiz-C. (eds). La Sierra Nevada de Santa Marta, Transecto Buritaca-La Cumbre. Estudios de ecosistemas tropandinos 2: 267-406 J. Cramer, Vaduz.

Cleef, A.M., Rangel-Ch., J.O. \& Salamanca-V., S. 2003. The andean rain forests of the parque Los Nevados transect, cordillera Central. In: T. Van der Hammen \& A. Dos-Santos (eds). Estudios de Ecosistemas Tropandinos. Ecoandes 5. J. Cramer (BORNTRAEGER) Berlín-Stuttgart.

Cortés-C., D.V. \& Rangel-Ch., J.O. 2011. Los bosques de mangle en un gradiente de salinidad en la bahía de Cispatá - Boca tinajones, departamento de Córdoba - Colombia. Caldasia 33 (1): 155-176.

Cortés-C., D.V. \& Rangel-Ch., J.O. 2012. Flora y estructura de la vegetación estuarina en la bahía de Cispatá-boca tinajones (Córdoba-Colombia). En: J.O. Rangel-Ch. (ed.). Colombia Diversidad Biótica XII. La región Caribe de Colombia: 539-573. Universidad Nacional de Colombia - Instituto de Ciencias Naturales.

Cuatrecasas, J. (1958). Aspectos de la vegetación natural de Colombia. Rev. Acad. Col. Ci. Ex. Fís. Nat. 10 (40): 221-268.
Diazgranados, M. (2012). A nomenclator for the frailejones (Espeletiinae Cuatrec., Asteraceae). Phytokeys 16: 1-52.

Dorr, J.L., Stergios, B., Smith, A. \& Cuello, N.L. 2000. Catalogue of the vascular plants of Guaramacal National Park, Portuguesa and Trujillo States, Venezuela. Smithsonian Institution. Contributions from the United States National Herbarium Volume 40: 1-155. Department of Botany, National Museum of Natural History. Washington D.C.

Dugand-G., A. 1970. Observaciones botánicas y geobotánicas en la costa colombiana del Caribe. Rev. Acad. Col. Cienc. 13 (52): 415-465.

Etter, A. 1997. Sabanas. En: M.E. Chaves \& N. Arango (eds). Informe nacional sobre el estado de la biodiversidad. Instituto de Investigación y de Recursos Biológicos Alexander von Humboldt, PNUMA, Ministerio del Medio Ambiente. Tomo I: 76-95 pp. Bogotá D.C.

Giraldo-Cañas., D. 2011. Catálogo de la familia Poaceae de Colombia. Darwiniana 49 (2): 139-147.

Hokche, O. \& Berry P. 2008. Análisis florístico. En: O. Hokche, P. Berry \& O. Huber (eds). Nuevo catálogo de la flora vascular de Venezuela. 69-110. Fundación Instituto Botánico de Venezuela. Fondo nacional de ciencia, tecnología e información. National Science Foundation NSF. Caracas, Venezuela.

Izco J. \& del Arco, M. 2003. Código Internacional de Nomenclatura Fitosociológica. Servicio de Publicaciones, Universidad de La Laguna. 156 pp. Santa Cruz de Tenerife.

Jorgensen, P.M. \& León-Y., S. (eds). 1999. Catálogo de las plantas vasculares del Ecuador. Monographs in Systematic Botany from the Missouri Botanical Garden 75: 43-106. Missouri Botanical Garden Press. St. Louis, Missouri.

La Marca, E. 2003a. Anfibios. En: M. Aguilera, A. Azócar \& E. González-J. (eds). Biodiversidad en Venezuela. 582595. Fundación Polar. Ministerio de ciencia y tecnología, FONACIT. Caracas, Venezuela.

La Marca, E. 2003b. Reptiles. En: M. Aguilera, A. Azócar \& E. González-J. (eds). Biodiversidad en Venezuela. 596608. Fundación Polar. Ministerio de ciencia y tecnología, FONACIT. Caracas, Venezuela.

Lozano-C., G. 1984. Comunidades vegetales del flanco Norte del cerro "El Cielo" y la flora vascular del Parque Nacional Natural Tayrona. En: T. Van der Hammen \& P.Ruiz (eds). La Sierra Nevada de Santa Marta (Colombia). Transecto Buritica-La Cumbre. Estudios de ecosistemas tropoandinos 2: 407-422. J. Cramer, Vaduz. Berlín.

Luteyn, J.L. 1999. Páramos a checklist of plant Diversity, geographical distribution, and botanical literatura. Mem. New York Bot. Gard. 84: 278 pp. New York.

Mann, O. 1964. Bases ecológicas para la explotación agrícola en América Latina. Monografías de la OEA. 2. Washington, D.C.

McCune, B. \& Mefford, M.J. 1999. PC-ORD. Multivariate analysis of ecological data. Version 4.41 (electronic manual). MjM Software. Gleneden Beach. EUA.

Ochoa-G., J. \& Aguilera M. 2003. Mamíferos. En: M. Aguilera, A. Azócar \& E. González-J. (eds). Biodiversidad en 
Venezuela. 650-672. Fundación Polar. Ministerio de ciencia y tecnología, FONACIT. Caracas, Venezuela.

Pinto-Z., J.H. 2010. La vegetación de la región de vida paramuna colombiana con especial referencia a la cordillera Occidental y los páramos del norte de Colombia. Tesis de Magíster Posgrado en Biología. Instituto de Ciencias Naturales, Universidad Nacional de Colombia. Bogotá D.C.

Pinto-Z., J.H. \& Rangel-Ch., J.O. 2010a. La vegetación de los páramos del norte de Colombia (Sierra Nevada de Santa Marta, Serranía de Perijá). En: J.O. Rangel-Ch. (ed.). Colombia Diversidad Biótica X: Cambios global (natural) y climático (antrópico) en el páramo colombiano: 289-410. Universidad Nacional de Colombia-Instituto de Ciencias Naturales. Bogotá D.C.

Pinto-Z., J.H. \& Rangel-Ch., J.O. 2010b. La vegetación paramuna de la cordillera Occidental colombiana I: las formaciones zonales. En: J.O. Rangel-Ch. (ed.). Colombia Diversidad Biótica X: Cambios global (natural) y climático (antrópico) en el páramo colombiano: 181-287. Universidad Nacional de Colombia-Instituto de Ciencias Naturales. Bogotá D.C.

Rangel-Ch., J.O., A.M. Cleef., T. Van der Hammen \& R. Jaramillo M. 1982. Tipos de vegetación en el transecto Buritica La Cumbre Sierra Nevada de Santa Marta (entre 0 y 4100 m) Colombia Geográfica 10 (1): 1-18. IGAC. Bogotá. ISSN: 01205366.

Rangel-Ch., J.O. \& Lozano-C., G. 1986. Un perfil de vegetación entre la Plata (Huila) y el Volcán del Puracé. Caldasia 14 (68-70): 503-547.

Rangel-Ch., J.O. 1995. (ed.) Colombia Diversidad Biótica I. Clima, Centros de concentración de especies, fauna. Instituto de Ciencias Naturales - Universidad Nacional de Colombia. 442 pp. Bogotá D.C.

Rangel-Ch., J.O. \& Velázquez, A. 1997. Métodos de estudio de la vegetación. En: J.O. Rangel-Ch., P. Lowy-C. \& M. Aguilar-P. Colombia Diversidad Biótica II. Instituto de Ciencias Naturales, Universidad Nacional de Colombia \& IDEAM: 59-87 pp. Bogotá D.C.

Rangel-Ch., J.O., Lowy-C., P., Aguilar-P., M. \& Garzón-C., A. 1997. Tipos de vegetación en Colombia. En: J.O. Rangel-Ch., P.Lowy-C. \& M. Aguilar-P. Colombia Diversidad Biótica II. Instituto de Ciencias Naturales, Universidad Nacional de Colombia \& IDEAM: 367-389 pp. Bogotá D.C.

Rangel-Ch., J.O. 2000a. (ed.). Colombia Diversidad Biótica III. La región de vida paramuna. Instituto de Ciencias Naturales - Instituto Alexander von Humboldt. 902 pp. Bogotá D.C.

Rangel-Ch., J.O. 2000b. Clima. En: J.O. Rangel-Ch. (ed.). Colombia Diversidad Biótica III. La región de vida paramuna. 85-125. Instituto de Ciencias Naturales-Instituto Alexander von Humboldt. Bogotá D.C.

Rangel-Ch., J.O. 2000c. La Diversidad Beta: Tipos de vegetación. En: J.O. Rangel-Ch. (ed.). Colombia Diversidad Biótica III. La región de vida paramuna. 658-719. Instituto de Ciencias Naturales-Instituto Alexander von Humboldt. Bogotá D.C.

Rangel-Ch., J.O. 2004a. (ed.). Colombia Diversidad Biótica IV. El Chocó biogeográfico/Costa Pacífica. Instituto de Ciencias Naturales - Conservación Internacional. 997 pp. Bogotá D.C.
Rangel-Ch., J.O. 2004b. Ecosistemas del Chocó biogeográfico: síntesis final. En: Rangel-Ch., J.O. (ed). Colombia Diversidad Biótica IV. El Chocó biogeográfico/Costa Pacífica: 937-976. Instituto de Ciencias Naturales. Bogotá, D.C.

Rangel-Ch., J.O. \& Arellano, H. 2004. Clima del Chocó biogeográfico. En: Rangel-Ch., J.O. (ed.). Colombia Diversidad Biótica IV. El Chocó biogeográfico/Costa Pacífica: 39-82. Instituto de Ciencias Naturales. Bogotá D.C.

Rangel-Ch., J.O., Cleef, A.M., Salamanca, S. \& Ariza, C.L. 2005b. La vegetación de los bosques y selvas del Tatamá. En: T. Van der Hammen, J.O. Rangel-Ch. \& A.M. Cleef (eds). Estudios de Ecosistemas Tropandinos-Ecoandes 6. La cordillera Occidental, transecto de Tatamá: 469-644. J. Cramer, (BORNTRAEGER) Berlín-Stuttgaart.

Rangel-Ch., J.O., M. Aguilar \& A. Florez. 2005c. Clima en el área del transecto Tatamá. En: T. Van der Hammen, J.O. Rangel-Ch. \& A.M. Cleef (eds). Estudios de Ecosistemas Tropandinos-Ecoandes 6. La cordillera Occidental, transecto de Tatamá: 47-104. J. Cramer, (BORNTRAEGER) BerlínStuttgaart. ISBN: 3-443-50028-5.

Rangel-Ch., J.O. 2005/2006. La biodiversidad de Colombia. Palimpsestos 5: 292-304.

Rangel-Ch., J.O. 2006. The biodiversity of the Colombian paramo region and its relation to antropogenic impact: 103-118. In: E. Spehn, M. Liebermann \& C. Korner (eds). Land use change and mountain biodiversity. CRC Press. Taylor \& Francis Group. Boca Raton.

Rangel-Ch., J.O. \& H. Arellano. 2008. El clima en el área del transecto Sumapaz (cordillera Oriental). En: T. Van der Hammen, J.O. Rangel-Ch. \& A.M. Cleef (eds). Estudios de Ecosistemas Tropandinos-Ecoandes 7. La cordillera Oriental, transecto de Sumapaz: 143-184. J. Cramer, (BORNTRAEGER) Berlín-Stuttgaart.

Rangel-Ch., J.O., Cleef, A.M. \& Arellano, H. 2008. La vegetación de los bosques y selvas del transecto del Sumapaz. En: T. Van der Hammen, J.O. Rangel-Ch. \& A.M. Cleef (eds). Estudios de Ecosistemas Tropandinos-Ecoandes 7. La cordillera Oriental, transecto de Sumapaz: 695-798. J. Cramer, (BORNTRAEGER) Berlín-Stuttgaart.

Rangel-Ch., J.O. 2009. (ed.). Colombia Diversidad Biótica VIII. Media y baja montaña de la serranía de Perijá. Universidad Nacional de Colombia-Instituto de Ciencias Naturales. 708 pp. Bogotá.

Rangel-Ch., J.O. \& H. Arellano-P. 2009. La vegetación de las selvas y los bosques de la serranía de Perijá. En: J.O. Rangel-Ch. (ed.). Colombia Diversidad Biótica VIII. Media y baja montaña de la serranía de Perijá: 245-298. Universidad Nacional de Colombia-Instituto de Ciencias Naturales-CORPOCESAR. Bogotá.

Rangel-Ch., J.O. \& Avella, A. 2011. Oak forests of Quercus humboldtii in the Caribbean region and distribution patterns related with environmental factors in Colombia. Plant Biosystems 145 (suppl 1): 186-198.

Rangel-Ch., J.O. 2012a. (ed.). Colombia Diversidad Biótica XII. La región Caribe de Colombia. Universidad Nacional de Colombia-Instituto de Ciencias Naturales. 1018 pp. Bogotá. 
Rangel-Ch., J.O. 2012b. La vegetación de la región Caribe de Colombia: composición florística y aspectos de la estructura. En: J.O. Rangel-Ch. (ed.). Colombia Diversidad Biótica XII. La región Caribe de Colombia: 365-476. Universidad Nacional de Colombia - Instituto de Ciencias Naturales. Bogotá D.C.

Rangel-Ch., J.O. 2012c. Ecosistemas del Caribe colombiano. En: J.O. Rangel-Ch. (ed.). Colombia Diversidad Biótica XII. La región Caribe de Colombia: 963-1009. Universidad Nacional de Colombia - Instituto de Ciencias Naturales. Bogotá D.C.

Rangel-Ch., J.O. \& Carvajal-C., J.E. 2012. Clima de la region Caribe colombiana. En: J.O. Rangel-Ch. (ed.). Colombia Diversidad Biótica XII. La región Caribe de Colombia: 67-129. Universidad Nacional de Colombia - Instituto de Ciencias Naturales. Bogotá D.C.

Rangel-Ch., J.O. \& Pinto-Z., J.H. 2012. Colombian Páramo Vegetation Database (CPVD) - the database on high Andean páramo vegetation in Colombia. Biodiversity \& Ecology 4: 275-286.

Rangel-Ch., J.O. 2014. Ecosistemas de la Orinoquia de Colombia. En: J.O. Rangel-Ch. (ed.) Colombia Diversidad Biótica XIV. La región de la Orinoquia de Colombia: 807-847. Instituto de Ciencias Naturales, Universidad Nacional de Colombia. Bogotá D.C.

Rangel-Ch., J.O. ed. 2014. Colombia Diversidad Biótica XIV. La región de la Orinoquia de Colombia. Universidad Nacional de Colombia-Instituto de Ciencias Naturales. 895 pp. Bogotá D.C.

Rangel-Ch., J.O. \& V. Minorta-C. 2014. Los tipos de vegetación de la Orinoquia colombiana. En: J.O. Rangel-Ch. (ed.). Colombia Diversidad Biótica XIV. La región de la Orinoquia de Colombia: 533-612. Universidad Nacional de Colombia - Instituto de Ciencias Naturales. Bogotá D.C.

Real Academia Española 2001. Diccionario de la lengua española. Fascículo 6. Madrid.

Rieger, W. (1976). Vegetations Kundliche untersuchungen auf Guajira-Halbinsel (Nordost-Kolumbien). Giess. Geogr. Schr. 40: 142 pp.

Rincón-E., A.J., J.O. Rangel-Ch. \& Aguirre-C., J. 2014. Musgos, líquenes, helechos y afines de la Orinoquia colombiana. En: J.O. Rangel-Ch. (ed.). Colombia Diversidad Biótica XIV. La región de la Orinoquia de Colombia: 419-446. Universidad Nacional de Colombia - Instituto de Ciencias Naturales. Bogotá D.C.

Rivera-D., O. \& Rangel-Ch., J.O. 2012. Diversidad de espermatofitos de la región Caribe colombiana. En: J.O. Rangel-Ch. (ed.). Colombia Diversidad Biótica XII. La región Caribe de Colombia: 199-317. Universidad Nacional de Colombia - Instituto de Ciencias Naturales. Bogotá D.C.

Roa, S. \& Ruiz-C., P. 1993. Anfibios. Contribución al Proyecto Estudio de la diversidad biótica de Colombia. Convenio Inderena-Universidad Nacional de Colombia (Documento interno). Bogotá D.C.
Ruiz-Carranza, P.M., Ardila-Robayo, M. C. \& Lynch, J.D. 1996. Lista actualizada de la fauna Amphibia de Colombia. Rev. Acad. Colomb. Cienc. Ex. Fis. Nat. 20 (77): 365-415.

Sánchez-P., H., Álvarez-L., R., Pinto-N., F., Sánchez-A., A., Renjifo, J.C., Garcia-H., I. \& Acosta-P., M.T. 1997. Diagnóstico y zonificación preliminar de los manglares del Caribe Colombiano. Ministerio del Medio Ambiente, OIMT. 511 pp. Bogotá D.C.

Sklenar, P., Luteyn, J.L., Ulloa-U., C., Jorgensen, P.M. \& Dillon, M.O. 2005. Flora génerica de los páramos. Guía ilustrada de las plantas vasculares. Memoirs of The New York Botanical Garden 92; 1-499.

Solari, S., Muñoz-S., Y., Rodríguez-M., J.V., Defler, T.R., Ramírez-C., H.E. \& Trujillo, F. (2013). Riqueza, endemismo y conservación de los mamíferos de Colombia. Mastozoología Neotropical 20 (2): 301-365. Mendoza.

Sturm, H. \& Rangel-Ch., J.O. 1985. Ecología de los páramos andinos. Una visión preliminar integrada. Biblioteca $\mathrm{J}$. Jerónimo Triana. Instituto de Ciencias Naturales. 250 pp. Bogotá D.C.

Trojer, H. (1958). Meteorología y Climatología de la vertiente del Pacífico Colombiano. Revista Acad. Colomb. Ci. Exact. 10 (40): 199-219.

Van der Hammen, T. 1984. Ecosistemas zonales en el flanco Norte de la Sierra Nevada se Santa Marta (transecto BuritacaLa Cumbre). En: T. Van der Hammen \& P.M. Ruiz (eds). La Sierra Nevada de Santa Marta (transecto Buritaca-La Cumbre). Estudio de ecosistemas tropandinos 2: 589-603. J. Cramer. Berlín-Stuttgart.

Van der Hammen, T. 2003. Ecosistemas zonales en los flancos oeste y este de la cordillera Central (transecto Parque los Nevados). En: T. Van der Hammen \& Alice G. Dos Santos (eds). La Cordillera Central Colombiana Transecto Parque los Nevados. Estudio de ecosistemas tropandinos 5: 503545. J. Cramer. Berlín-Stuttgart.

Van der Hammen, T. 2005. Zonal ecosystems of the west and east flanks of the Colombian Western Cordillera (Tatamá transect). En: T. Van der Hammen., J. Orlando Rangel \& A.M. Cleef. (eds). La Cordillera Occidental Colombiana Transecto Tatamá. Estudio de ecosistemas tropandinos 6: 935-972. J. Cramer. Berlín-Stuttgart.

Van der Hammen, T. 2008. Zonal ecosystems of the west and east flanks of the Colombian eastern t Cordillera (Sumapaz transect). En: T. Van der Hammen (ed.). La Cordillera Occidental Colombiana Transecto Tatamá. Estudio de ecosistemas tropandinos 6: 935-972. J. Cramer. BerlínStuttgart.

Vargas, G. \& J.J. Sánchez. 2005. Plantas con flores de los páramos de Costa Rica y Panamá: el páramo ístmico. En: M. Kappelle \& S.P. Horn (eds). Páramos de Costa Rica: 397 -436. INBio, Ministerio del Ambiente y Energía, Wotro, The Nature Conservancy. San José de Costa Rica.

Zuluaga-R., S. 1987. Observaciones fotoecológicas en el Darién colombiano. Perez-Arbelaezia 1 (4-5): 86-145. 